\title{
Stochastic averaging for the non-autonomous mixed stochastic differential equations with locally Lipschitz coefficients
}

\author{
Ruifang Wang ${ }^{\mathrm{a}}$, Yong $\mathrm{Xu}^{\mathrm{a}, \mathrm{b}, *}$, Hongge Yue \\ ${ }^{a}$ School of Mathematics and Statistics, Northwestern Polytechnical University, Xi'an, 710072, China \\ ${ }^{b}$ MIIT Key Laboratory of Dynamics and Control of Complex Systems, Northwestern Polytechnical \\ University, Xi'an, 710072, China
}

\begin{abstract}
This paper investigates a non-autonomous slow-fast system, which is generalized by stochastic differential equations (SDEs) with locally Lipschitz coefficients, subjected to standard Brownian motion (Bm) and fractional Brownian motion (fBm) with Hurst parameter $1 / 2<$ $H<1$. We concentrate on how to handle both types of integrals with respect to $\mathrm{Bm}$ and $\mathrm{fBm}$ and the locally Lispchitz continuity. The pathwise approach and the Itô stochastic calculus are combined with the technique of stopping time to establish the averaging principle where the averaged equation is defined. Then, the slow component of the original slow-fast system converges to the solution of the proposed averaged equation in the mean square sense is verified.
\end{abstract}

Keywords. Averaging principle, fractional Brownian motion, non-autonomous system, generalised Riemann-Stieltjes integral, Itô stochastic integral

Mathematics subject classification. 60G22, 60H10, 34C29, 37B55

\section{Introduction}

In this paper, we study the following SDEs driven by fBm and standard Bm:

$$
\left\{\begin{array}{lr}
d u_{t}^{\epsilon}=b_{1}\left(t, u_{t}^{\epsilon}, v_{t}^{\epsilon}\right) d t+f_{1}\left(t, u_{t}^{\epsilon}\right) d W_{t}^{1}+g_{1}\left(t, u_{t}^{\epsilon}\right) d B_{t}^{H}, & u_{0}^{\epsilon}=x \in \mathbb{R}^{n}, \\
d v_{t}^{\epsilon}=\frac{1}{\epsilon} b_{2}\left(t, u_{t}^{\epsilon}, v_{t}^{\epsilon}\right) d t+\frac{1}{\sqrt{\epsilon}} f_{2}\left(t, u_{t}^{\epsilon}, v_{t}^{\epsilon}\right) d W_{t}^{2}, & v_{0}^{\epsilon}=y \in \mathbb{R}^{m}
\end{array}\right.
$$

where $t \in[0, T]$ and $\epsilon \in(0,1]$ is a small positive parameter which represents the ratio of the natural time scale between the slow variable $u_{t}^{\epsilon} \in \mathbb{R}^{n}$ and fast variable $v_{t}^{\epsilon} \in \mathbb{R}^{m}$. Moreover, $B^{H}=\left\{B_{t}^{H}, t \in[0, T]\right\}(H \in(1 / 2,1))$ is $d_{1}$-dimensional $\mathrm{fBm}, W^{1}=\left\{W_{t}^{1}, t \in[0, T]\right\}$ and $W^{2}=\left\{W_{t}^{2}, t \in[0, T]\right\}$ are $d_{2}$ and $d_{3}$-dimensional standard Bm, respectively. Assume that $W^{1}, W^{2}$ and $B^{H}$ are mutually independent processes, and initial variables $x, y$ are fixed and independent of $\left(W^{1}, W^{2}, B^{H}\right)$.

\footnotetext{
${ }^{*}$ Corresponding author

Email addresses: wrfjy@yahoo.com (Ruifang Wang), hsux3@nwpu.edu.cn (Yong Xu), yuehongge8030163.com (Hongge Yue)
} 
Recall that fBm with Hurst index $H \in(0,1)$ is a zero mean Gaussian process $\left\{B_{t}^{H}, t \geq 0\right\}$ with covariance function

$$
R_{H}(s, t)=\frac{1}{2}\left(s^{2 H}+t^{2 H}-|t-s|^{2 H}\right) .
$$

Notice that $B^{H}$ is a standard $\mathrm{Bm}$ if $H=1 / 2$, but if $H \neq 1 / 2$, it does not have independent increments. Moreover, from (1.2) we deduce that, $\mathbb{E}\left|B_{t}-B_{s}\right|^{2}=|t-s|^{2 H}$. As a consequence, the process $B^{H}$ has $\alpha$-Hölder continuous paths for all $\alpha \in(0, H)$. Kolmogorov introduced the process $B^{H}$ [1] in 1940, and Mandelbrot and Van Ness [2] later named it as fBm in 1968.

The averaging principle is a kind of approximate theorems to simplify stochastic dynamical systems with different time-scales, and the first related result about stochastic case was studied by Khasminskii [3] in 1968. Since then, the averaging principle has been investigated by a number of investigators. For instance, in the case of the autonomous system: Givon [4], Freidlin and Wentzell [5], Duan [6], Xu and his co-workers [7, 8] studied the averaging principle of SDEs. In addition, the averaging principle of stochastic partial differential equations (SPDEs) also have been investigated by Cerrai [9, 10], Wang and Roberts [11], Pei and Xu $[12,13]$ and others in recent years. For the non-autonomous case, Cerrai [14] and Liu [15] studied the averaging principle for non-autonomous slow-fast systems driven by Brownian motion, which is generalized by SPDEs and SDEs respectively. Moreover, Xu [16] also studied a class of non-autonomous slow-fast systems of SPDEs driven by Gaussian noises and Poisson random measures, and proved the averaging principle in the sense of probability. However, either Gaussian noises or Poisson random measures can not describe the disturbances with long-range dependence. This paper is to consider a class of non-autonomous slow-fast system of SDEs driven by standard Bm and fBm, and the convergence of averaging principle in the mean square sense is to be proved.

First, the existence and uniqueness of solutions for (1.1) is studied. Comparing with the work of Guerra and Nualart [17], the conditional expectation (given $x, y$ and $B^{H}$ ) in [17] is extended to general expectation on $\left(\Omega, \mathcal{F},\left\{\mathcal{F}_{t}\right\}_{t \geq 0}, \mathbb{P}\right.$ ) (where $\left\{\mathcal{F}_{t}\right\}_{t \geq 0}$ is the $\sigma$-field generated by the random variables $W_{t}^{1}, W_{t}^{2}, B_{t}^{H}$ and the $\mathbb{P}$-null sets) in our work and the coefficients are assumed to satisfy locally Lipschitz conditions. To overcome the problem of $B^{H}$ is not a semimartingale if $H \neq 1 / 2$, we interpret the integral $\int_{0}^{t} f_{1}\left(r, u_{r}^{\epsilon}\right) d W_{r}^{1}, \int_{0}^{t} f_{2}\left(r, u_{r}^{\epsilon}, v_{r}^{\epsilon}\right) d W_{r}^{2}$ as an Itô stochastic integral and the integral $\int_{0}^{t} g_{1}\left(r, u_{r}^{\epsilon}\right) d B_{r}^{H}$ as a generalised RiemannStieltjes integral in the sense of Zähle [18] in our work. Then, the Itô stochastic calculus and the pathwise approach are combined to handle these two kinds of integrals and the Garsia-Rodemich-Rumsey inequality is used to overcome the problem of $B^{H}$ will produce some higher order terms. Moreover, the technique of stopping time is also used to deal with the problem of expectation and locally Lispchitz continuity.

Then, consider that the coefficients in equation (1.1) depend on time, the corresponding equation associated to the fast equation by fixed $s>0$ and frozen slow component $x \in \mathbb{R}^{n}$

$$
d v_{t}=b_{2}\left(s, x, v_{t}\right) d t+f_{2}\left(s, x, v_{t}\right) d W_{t}^{2}, \quad v_{0}=y \in \mathbb{R}^{m}
$$

is introduced. Further, the existence of the unique invariant measure $\mu^{s, x}$ for the equation 
(1.3) is proved and the averaged coefficient can be defined as

$$
\bar{b}_{1}(s, x)=\int_{\mathbb{R}^{m}} b_{1}(s, x, z) \mu^{s, x}(d z) .
$$

Finally, the detailed proof of the convergence result is presented by using the technique of time discretization and truncation. That is, under some reasonable assumptions, the purpose of this paper is to show the convergence of averaging principle in the mean square sense:

$$
\lim _{\epsilon \rightarrow 0} \sup _{t \in[0, T]} \mathbb{E}\left\|u_{t}^{\epsilon}-\bar{u}_{t}\right\|_{\alpha}^{2}=0
$$

where $\bar{u}_{t}$ is the solution to the so-called averaged equation as:

$$
d \bar{u}_{t}=\bar{b}_{1}\left(t, \bar{u}_{t}\right) d t+f_{1}\left(t, \bar{u}_{t}\right) d W_{t}^{1}+g_{1}\left(t, \bar{u}_{t}\right) d B_{t}^{H}, \quad \bar{u}_{0}=x \in \mathbb{R}^{n} .
$$

This paper is organized as follows, in Section 2, some notations and assumptions which will be used in the analysis of equation (1.1) is introduced and the main results is claimed. Section 3 is committed to proving the existence and uniqueness of solutions to 1.1. In Section 4, the averaging principle is obtained by using the generalized Khasminskii method where the averaged equation is defined. Note that, $C>0$ with or without subscripts represents a general constant, the value of which may vary for different cases in this paper.

\section{Preliminaries, assumptions and main result}

Now, we recall some definitions and results that will be used throughout the paper. Let $|\cdot|$ be the Euclidean norm, $\langle\cdot, \cdot\rangle$ be the Euclidean inner product and $\|\cdot\|$ be the matrix norm.

Let $1 / 2<H<1,1-H<\alpha<1 / 2$ and $k, l \in N^{+}$, denote $W_{0}^{\alpha, \infty}\left(0, T ; \mathbb{R}^{k}\right)$ the space of measurable functions $f:[0, T] \rightarrow \mathbb{R}^{k}$ such that

$$
\|f\|_{\alpha, \infty}:=\sup _{t \in[0, T]}\|f(t)\|_{\alpha}<\infty
$$

where

$$
\|f(t)\|_{\alpha}:=|f(t)|+\int_{0}^{t} \frac{|f(t)-f(s)|}{(t-s)^{\alpha+1}} d s .
$$

For $0<\eta \leq 1$, let $C^{\eta}\left(0, T ; \mathbb{R}^{k}\right)$ be space of $\eta$-Hölder continuous functions $f:[0, T] \rightarrow \mathbb{R}^{k}$, equipped with the norm

$$
\|f\|_{\eta}:=\sup _{t \in[0, T]}|f(t)|+\sup _{0 \leq s<t \leq T} \frac{|f(t)-f(s)|}{(t-s)^{\eta}}<\infty .
$$

Denote by $W_{T}^{1-\alpha, \infty}\left(0, T ; \mathbb{R}^{k}\right)$ the space of measurable functions $g:[0, T] \rightarrow \mathbb{R}^{k}$ such that

$$
\|g\|_{1-\alpha, \infty, T}:=\sup _{0<s<t<T}\left(\frac{|g(t)-g(s)|}{(t-s)^{1-\alpha}}+\int_{s}^{t} \frac{|g(r)-g(s)|}{(r-s)^{2-\alpha}} d r\right)<\infty .
$$


Moreover, denote by $W_{0}^{\alpha, 1}\left(0, T ; \mathbb{R}^{l}\right)$ the space of measurable functions $f:[0, T] \rightarrow \mathbb{R}^{l}$ such that

$$
\|f\|_{\alpha, 1}:=\int_{0}^{T} \frac{|f(s)|}{s^{\alpha}} d s+\int_{0}^{T} \int_{0}^{s} \frac{|f(s)-f(r)|}{(s-r)^{\alpha+1}} d r d s<\infty .
$$

Then, if $f \in W_{0}^{\alpha, 1}\left(0, T ; \mathbb{R}^{l}\right)$ and $g \in W_{T}^{1-\alpha, \infty}\left(0, T ; \mathbb{R}^{k}\right)$, for any $t \in[0, T]$, we know that $\int_{0}^{t} f d g$ exists, and have

$$
\left|\int_{0}^{t} f \mathrm{~d} g\right| \leq \Lambda_{\alpha}^{0, t}(g)\|f\|_{\alpha, 1}
$$

where

$$
\Lambda_{\alpha}^{0, T}(g):=\frac{1}{\Gamma(1-\alpha)} \sup _{0<s<t<T}\left|\left(D_{t-}^{1-\alpha} g_{t-}\right)(s)\right| \leq \frac{1}{\Gamma(1-\alpha) \Gamma(\alpha)}\|g\|_{1-\alpha, \infty, T}<\infty,
$$

and $\left(D_{t-}^{1-\alpha} g_{t-}\right)(s)$ is the Weyl derivatives [19] of $g$.

Remark 2.1. In particular, the trajectories of $f B m B^{H}(H>1 / 2)$ belong to $W_{T}^{1-\alpha, \infty}\left(0, T ; \mathbb{R}^{k}\right)$ where $(1-H<\alpha<1 / 2)$. As a consequence, if the trajectories of a stochastic process $u=$ $\left\{u_{t}, t \in[0, T]\right\}$ belong to the space $W_{0}^{\alpha, 1}\left(0, T ; \mathbb{R}^{l}\right)$, the generalised Riemann-Stieltjes integrals $\int_{0}^{T} u_{s} d B_{s}^{H}$ exists, and the following estimate holds

$$
\left|\int_{0}^{T} u_{s} d B_{s}^{H}\right| \leq \Lambda_{\alpha}^{0, T}\left(B^{H}\right)\|u\|_{\alpha, 1}
$$

where $\Lambda_{\alpha}^{0, T}\left(B^{H}\right):=\frac{1}{\Gamma(1-\alpha)} \sup _{0<s<t<T}\left|\left(D_{t-}^{1-\alpha} B_{t-}^{H}\right)(s)\right|$ has moments of all orders (see Lemma 7.5 in Nualart and Răşcanu [20]).

The following lemma is the so-called Garsia-Rodemich-Rumsey inequality (see Theorem 1.4 in [21]):

Lemma 2.2. For any $p \geq 1$ and $\theta>p^{-1}$, there exists some constant $C_{\theta, p}>0$ such that for any continuous function $f$ on $[0, T]$, have

$$
|f(t)-f(s)|^{p} \leq C_{\theta, p}|t-s|^{\theta p-1} \int_{0}^{T} \int_{0}^{T} \frac{|f(x)-f(y)|^{p}}{|x-y|^{\theta p+1}} d x d y .
$$

In this paper, the following maps

$$
\begin{aligned}
& b_{1}:[0, \infty) \times \mathbb{R}^{n} \times \mathbb{R}^{m} \times \Omega \rightarrow \mathbb{R}^{n} ; \\
& f_{1}:[0, \infty) \times \mathbb{R}^{n} \times \Omega \rightarrow \mathbb{R}^{n \times d_{2}} ; \\
& g_{1}:[0, \infty) \times \mathbb{R}^{n} \times \Omega \rightarrow \mathbb{R}^{n \times d_{1}} ; \\
& b_{2}:[0, \infty) \times \mathbb{R}^{n} \times \mathbb{R}^{m} \times \Omega \rightarrow \mathbb{R}^{m} ; \\
& f_{2}:[0, \infty) \times \mathbb{R}^{n} \times \mathbb{R}^{m} \times \Omega \rightarrow \mathbb{R}^{m \times d_{3}}
\end{aligned}
$$

are continuous. Then, the following assumptions are supposed to hold for $\mathbb{P}$-almost all $\omega \in \Omega:$ 
(A1) (a) For any $R \in \mathbb{R}, y \in \mathbb{R}^{m}$ and $x_{i} \in \mathbb{R}^{n}(i=1,2)$ with $\left|x_{i}\right| \leq R$, there exist some constants $\theta_{1} \geq 0$, such that

$$
\left|b_{1}\left(t, x_{1}, y\right)-b_{1}\left(t, x_{2}, y\right)\right|+\left\|f_{1}\left(t, x_{1}\right)-f_{1}\left(t, x_{2}\right)\right\| \leq C_{R, T}\left(1+|y|^{\theta_{1}}\right)\left|x_{1}-x_{2}\right| .
$$

(b) For any $x \in \mathbb{R}^{n}$ and $y_{1}, y_{2} \in \mathbb{R}^{m}$, there exist some constants $\theta_{2}, \theta_{3} \geq 0$ and $0<\kappa \leq 1$, such that

$$
\begin{gathered}
\left|b_{1}\left(t, x, y_{1}\right)-b_{1}\left(t, x, y_{2}\right)\right| \leq C_{T}\left|y_{1}-y_{2}\right|\left(1+|x|^{\theta_{2}}+\left|y_{1}\right|^{\theta_{3}}+\left|y_{2}\right|^{\theta_{3}}\right) ; \\
\left|b_{1}\left(t, x_{1}, y_{1}\right)-b_{1}\left(s, x_{1}, y_{1}\right)\right|+\left\|f_{1}\left(t, x_{1}\right)-f_{1}\left(s, x_{1}\right)\right\| \leq C_{T}|t-s|^{\kappa}\left(1+\left|x_{1}\right|^{\theta_{2}}+|y|^{\theta_{3}}\right) ; \\
\left|b_{1}\left(t, x_{1}, y_{1}\right)\right|+\left\|f_{1}\left(t, x_{1}\right)\right\| \leq C_{T}\left(1+\left|x_{1}\right|+\left|y_{1}\right|\right) .
\end{gathered}
$$

(A2) (a) The mapping $g_{1}$ is continuously differentiable in $x \in \mathbb{R}^{n}$. For any $R \in \mathbb{R}$ and $x_{i} \in \mathbb{R}^{n}(i=1,2)$ with $\left|x_{i}\right| \leq R$, there exist some constants $0<\gamma \leq 1$, such that

$$
\begin{gathered}
\left\|g_{1}\left(t, x_{1}\right)-g_{1}\left(t, x_{2}\right)\right\| \leq C_{R, T}\left|x_{1}-x_{2}\right| ; \\
\left\|\nabla_{x_{1}} g_{1}\left(t, x_{1}\right)-\nabla_{x_{2}} g_{1}\left(t, x_{2}\right)\right\| \leq C_{R, T}\left|x_{1}-x_{2}\right|^{\gamma},
\end{gathered}
$$

where $\nabla_{x}$ is the standard gradient with respect to the variable $x$.

(b) For any $x \in \mathbb{R}^{n}$ and $s, t \in[0, T]$, there exist some constants $0<\beta \leq 1$, such that

$$
\begin{gathered}
\left\|g_{1}(t, x)\right\| \leq C_{T}(1+|x|) ; \\
\left\|\nabla_{x} g_{1}(t, x)-\nabla_{x} g_{1}(s, x)\right\|+\left\|g_{1}(t, x)-g_{1}(s, x)\right\| \leq C_{T}|t-s|^{\beta} .
\end{gathered}
$$

(A3) (a) For any $x \in \mathbb{R}^{n}$ and $t \in[0, T]$, the mapping $b_{2}(t, x, \cdot)$ is locally Lipschitz continuous and $f_{2}(t, \cdot, \cdot)$ is Lipschitz continuous.

(b) For any $x_{i} \in \mathbb{R}^{n}, y_{i} \in \mathbb{R}^{m}$ and $t \in[0, T]$, there exist some constants $\alpha_{1}, \alpha_{2}>0$ and $\iota \in(0,1]$, such that

$$
\begin{gathered}
\left|b_{2}\left(t, x_{1}, y_{1}\right)-b_{2}\left(t, x_{2}, y_{1}\right)\right| \leq C_{T}\left|x_{1}-x_{2}\right|\left(1+\left|x_{1}\right|^{\alpha_{1}}+\left|x_{2}\right|^{\alpha_{1}}+\left|y_{1}\right|^{\alpha_{2}}\right) ; \\
\left|b_{2}\left(t, x_{1}, y_{1}\right)-b_{2}\left(s, x_{1}, y_{1}\right)\right|+\left|f_{2}\left(t, x_{1}, y_{1}\right)-f_{2}\left(s, x_{1}, y_{1}\right)\right| \leq C_{T}|t-s|^{\iota}\left(1+\left|x_{1}\right|^{\alpha_{1}}+\left|y_{1}\right|^{\alpha_{2}}\right) \\
\left|b_{2}\left(t, x_{1}, y_{1}\right)\right|+\left\|f_{2}\left(t, x_{1}, y_{1}\right)\right\| \leq C_{T}\left(1+\left|x_{1}\right|+\left|y_{1}\right|\right) .
\end{gathered}
$$

(A4) Assume that for any $t \in \mathbb{R}, x \in \mathbb{R}^{n}$ and $y \in \mathbb{R}^{m}, b_{1}(t, x, y)$ and $f_{1}(t, x)$ are bounded.

(A5) (Strict monotonicity condition:) For any $t \in[0,+\infty), x \in \mathbb{R}^{n}, y_{1}, y_{2} \in \mathbb{R}^{m}$, there exist constants $\beta_{1}>0$, such that

$$
2\left\langle y_{1}-y_{2}, b_{2}\left(t, x, y_{1}\right)-b_{2}\left(t, x, y_{2}\right)\right\rangle+\left\|f_{2}\left(t, x, y_{1}\right)-f_{2}\left(t, x, y_{2}\right)\right\|^{2} \leq-\beta_{1}\left|y_{1}-y_{2}\right|^{2} .
$$

(Strict coercivity condition:) For some fixed $p \geq 2$, any $t \in[0,+\infty), x \in \mathbb{R}^{n}$ and $y \in \mathbb{R}^{m}$, there exist constants $C_{p, T}, \beta_{p}>0$, such that

$$
2\left\langle y, b_{2}(t, x, y)\right\rangle+(p-1)\left\|f_{2}(t, x, y)\right\|^{2} \leq-\beta_{p}|y|^{2}+C_{p, T}\left(1+|x|^{2}\right) .
$$


Remark 2.3. The existence and uniqueness of solutions for original equation (1.1) are guaranteed by the assumptions (A1)-(A4). Strict monotonicity condition guarantee the exponential ergodicity (see Lemma 4.5 in Section 4) holds and strict coercivity condition is used to ensures the existence of invariant measures for the frozen equation (see Lemma 4.1 in Section 4).

Under the above assumptions, the main result of this paper is claimed as follows:

Theorem 2.4. Assume that the conditions (A1)-(A4) hold. Then, for any $\alpha \in\left(1-H, \frac{1}{2} \wedge \beta\right.$ $\left.\wedge \frac{\gamma}{2}\right)$ and $\epsilon \in(0,1]$, there exists a unique solution $\left(u_{t}^{\epsilon}, v_{t}^{\epsilon}\right) \in\left(W_{0}^{\alpha, \infty}\left(0, T ; \mathbb{R}^{n}\right), W_{0}^{\alpha, \infty}\left(0, T ; \mathbb{R}^{m}\right)\right)$ of equation (1.1).

Theorem 2.5. Assume that the conditions (A1)-(A5) hold. Then, for any $\epsilon \in(0,1]$ and $\alpha \in\left(1-H, \frac{1}{2} \wedge \beta \wedge \frac{\gamma}{2}\right)$, we have

$$
\lim _{\epsilon \rightarrow 0} \sup _{t \in[0, T]} \mathbb{E}\left\|u_{t}^{\epsilon}-\bar{u}_{t}\right\|_{\alpha}^{2}=0
$$

where $\bar{u}_{t}$ is the solution of the corresponding averaged equation (1.5).

\section{Existence, uniqueness of the solutions}

In this section, we aim to prove the existence and uniqueness of solutions for a class of mixed SDEs (1.1) driven by fBm and Bm (Theorem 2.4). An auxiliary equation is introduced at first. Then, some estimates for this auxiliary equation are presented. Finally, the existence and uniqueness of solutions for original equation (1.1) is proved by defining the stopping time.

\subsection{Some a-priori estimates of $\left(u_{t}^{\epsilon, k}, v_{t}^{\epsilon, k}\right)$}

For any $k \in \mathbb{N}$, we define the following stopping time

$$
\tau_{k}:=\inf \left\{t \geq 0: \Lambda_{\alpha}^{0, t}\left(B^{H}\right) \geq k\right\},
$$

and study the following equation:

$$
\left\{\begin{array}{lrl}
d u_{t}^{\epsilon}=b_{1, k}\left(t, u_{t}^{\epsilon}, v_{t}^{\epsilon}\right) d t+f_{1, k}\left(t, u_{t}^{\epsilon}\right) d W_{t}^{1}+g_{1, k}\left(t, u_{t}^{\epsilon}\right) d B_{t}^{H, k}, & u_{0}^{\epsilon}=x \in \mathbb{R}^{n}, \\
d v_{t}^{\epsilon}=\frac{1}{\epsilon} b_{2, k}\left(t, u_{t}^{\epsilon}, v_{t}^{\epsilon}\right) d t+\frac{1}{\sqrt{\epsilon}} f_{2}\left(t, u_{t}^{\epsilon}, v_{t}^{\epsilon}\right) d W_{t}^{2}, & v_{0}^{\epsilon}=y \in \mathbb{R}^{m}
\end{array}\right.
$$

where $B_{t}^{H, k}=B_{t \wedge \tau_{k}}^{H}$ and

$$
b_{i, k}(t, x, y)= \begin{cases}b_{i}(t, x, y), & |x| \leq k \text { and }|y| \leq k, \\ b_{i}(t, x, y k /|y|), & |x| \leq k \text { and }|y|>k, \\ b_{i}(t, x k /|x|, y), & |x|>k \text { and }|y| \leq k \\ b_{i}(t, x k /|x|, y k /|y|), & |x|>k \text { and }|y|>k\end{cases}
$$

and

$$
f_{1, k}(t, x)=\left\{\begin{array}{ll}
f_{1}(t, x), & |x| \leq k, \\
f_{1}(t, x k /|x|), & |x|>k,
\end{array} \quad g_{1, k}(t, x)= \begin{cases}g_{1}(t, x), & |x| \leq k \\
g_{1}(t, x k /|x|), & |x|>k\end{cases}\right.
$$


It is easy to know that the mapping $b_{i, k}(t, \cdot, \cdot), f_{1, k}(t, \cdot)$ and $g_{1, k}(t, \cdot)$ are Lipschitz continuous and satisfy all conditions in (A1)-(A3). Moreover, for any $l>k$, we also have

$$
|x| \leq k \text { and }|y| \leq k \Rightarrow\left\{\begin{array}{l}
b_{i, k}(t, x, y)=b_{i, l}(t, x, y)=b_{i}(t, x, y) \\
f_{1, k}(t, x)=f_{1, l}(t, x)=f_{1}(t, x) \\
g_{1, k}(t, x)=g_{1, l}(t, x)=g_{1}(t, x)
\end{array}\right.
$$

and

$$
\Lambda_{\alpha}^{0, t}\left(B^{H}\right) \leq k \Rightarrow B_{t}^{H, k}=B_{t}^{H, l}=B_{t}^{H} .
$$

Then, using the same argument as [17], it is easy to get that for any fixed $k \in \mathbb{N}$, there exists a unique strong solution $\left(u_{t}^{\epsilon, k}, v_{t}^{\epsilon, k}\right) \in\left(W_{0}^{\alpha, \infty}\left(0, T ; \mathbb{R}^{n}\right), W_{0}^{\alpha, \infty}\left(0, T ; \mathbb{R}^{m}\right)\right)$ to equation (3.2).

Lemma 3.1. Under the assumptions (A1)-(A4), for any $\alpha \in\left(1-H, \frac{1}{2} \wedge \beta \wedge \frac{\gamma}{2}\right), p \geq 1$ and fixed $\epsilon_{0} \in(0,1]$, there exists some positive constant, such that

$$
\mathbb{E}\left\|u^{\epsilon_{0}, k}\right\|_{\alpha, \infty}^{p} \leq C_{\alpha, p, x, T}
$$

and

$$
\mathbb{E}\left\|v^{\epsilon_{0}, k}\right\|_{\alpha, \infty}^{p} \leq C_{\alpha, p, \epsilon_{0}, x, y, T}
$$

Proof: First, we estimate $\mathbb{E}\left\|u^{\epsilon_{0}, k}\right\|_{\alpha, \infty}^{p}$. For brevity, we denote

$$
\Psi_{t}^{\epsilon_{0}}\left(\lambda, u^{\epsilon_{0}, k}\right)=\sup _{r \in[0, t]} e^{-\lambda r}\left|u_{r}^{\epsilon_{0}, k}\right|,
$$

and

$$
\Phi_{t}^{\epsilon_{0}}\left(\lambda, u^{\epsilon_{0}, k}\right)=\sup _{r \in[0, t]} e^{-\lambda r} \int_{0}^{r} \frac{\left|u_{r}^{\epsilon_{0}, k}-u_{s}^{\epsilon_{0}, k}\right|}{(r-s)^{\alpha+1}} d s .
$$

In order to estimate $\left\|u^{\epsilon_{0}, k}\right\|_{\alpha, \infty}$, we first estimate $\Psi_{t}^{\epsilon_{0}}\left(\lambda, u^{\epsilon_{0}, k}\right)$. Thanks to the assumption (A4) and (2.1), it yields

$$
\begin{gathered}
\Psi_{t}^{\epsilon_{0}}\left(\lambda, u^{\epsilon_{0}, k}\right) \leq C_{x, T}\left(1+\sup _{r \in[0, t]}\left|\int_{0}^{r} f_{1, k}\left(s, u_{s}^{\epsilon_{0}, k}\right) d W_{s}^{1}\right|\right. \\
\left.+\Lambda_{\alpha}^{0, t}\left(B^{H, k}\right) \sup _{r \in[0, t]} \int_{0}^{r} e^{-\lambda(r-s)}\left(s^{-\alpha} \Psi_{s}^{\epsilon_{0}}\left(\lambda, u^{\epsilon_{0}, k}\right)+\Phi_{s}^{\epsilon_{0}}\left(\lambda, u^{\epsilon_{0}, k}\right)\right) d s\right) \\
\leq C_{x, T}\left(1+\Lambda_{\alpha}^{0, t}\left(B^{H, k}\right)\right)\left(1+\lambda^{\alpha-1} \Psi_{t}^{\epsilon_{0}}\left(\lambda, u^{\epsilon_{0}, k}\right)+\lambda^{-1} \Phi_{t}^{\epsilon_{0}}\left(\lambda, u^{\epsilon_{0}, k}\right)\right. \\
\left.\quad+\sup _{r \in[0, t]}\left|\int_{0}^{r} f_{1, k}\left(s, u_{s}^{\epsilon_{0}, k}\right) d W_{s}^{1}\right|\right)
\end{gathered}
$$

where the last equation used the following estimate [20, page 66]

$$
\int_{0}^{t} e^{-\lambda(t-r)} r^{-\alpha} d r \leq C \lambda^{\alpha-1}
$$


Then, we estimate $\Phi_{t}^{\epsilon_{0}}\left(\lambda, u^{\epsilon_{0}, k}\right)$. According to the equation (4.17) in [20], we can get

$$
\begin{gathered}
\Phi_{t}^{\epsilon_{0}}\left(\lambda, u^{\epsilon_{0}, k}\right) \leq C_{\alpha, x, T}\left(1+\sup _{r \in[0, t]} \int_{0}^{r} \frac{\left|\int_{s}^{r} f_{1, k}\left(\sigma, u_{\sigma}^{\epsilon_{0}, k}\right) d W_{\sigma}^{1}\right|}{(r-s)^{\alpha+1}} d s+\Lambda_{\alpha}^{0, t}\left(B^{H, k}\right) \sup _{r \in[0, t]} e^{-\lambda r}\right. \\
\left.\times\left(\int_{0}^{r} \frac{\left|g_{1, k}\left(s, u_{s}^{\epsilon_{0}, k}\right)\right|}{(r-s)^{2 \alpha}} d s+\int_{0}^{r} \int_{0}^{s}(r-\sigma)^{-\alpha} \frac{\left|g_{1, k}\left(s, u_{s}^{\epsilon_{0}, k}\right)-g_{1, k}\left(\sigma, u_{\sigma}^{\epsilon_{0}, k}\right)\right|}{(s-\sigma)^{1+\alpha}} d \sigma d s\right)\right) \\
\leq C_{\alpha, x, T}\left(1+\Lambda_{\alpha}^{0, t}\left(B^{H, k}\right)\right)\left(1+\sup _{r \in[0, t]} \int_{0}^{r}(r-s)^{-\alpha-1}\left|\int_{s}^{r} f_{1, k}\left(\sigma, u_{\sigma}^{\epsilon_{0}, k}\right) d W_{\sigma}^{1}\right| d s\right. \\
\left.+\lambda^{2 \alpha-1} \Psi_{t}^{\epsilon_{0}}\left(\lambda, u^{\epsilon_{0}, k}\right)+\lambda^{\alpha-1} \Phi_{t}^{\epsilon_{0}}\left(\lambda, u^{\epsilon_{0}, k}\right)\right),(3.10)
\end{gathered}
$$

where the last equation used the estimate [20, page 66]

$$
\int_{0}^{r} e^{-\lambda(r-s)}(r-s)^{-\alpha} d s=\Gamma(1-\alpha) \lambda^{\alpha-1}
$$

Let $C:=C_{x, T} \vee C_{\alpha, x, T}$ and $\lambda=\left(4 C\left(1+\Lambda_{\alpha}^{0, t}\left(B^{H, k}\right)\right)\right)^{\frac{1}{1-\alpha}}$. Combining (3.9) and (3.10), making simple transformations as [22], it is easy to get that

$$
\Psi_{t}^{\epsilon_{0}}\left(\lambda, u^{\epsilon_{0}, k}\right)+\Phi_{t}^{\epsilon_{0}}\left(\lambda, u^{\epsilon_{0}, k}\right) \leq C_{\alpha, x, T}\left(1+\Lambda_{\alpha}^{0, t}\left(B^{H, k}\right)\right)^{\frac{1}{1-\alpha}}\left(1+\Upsilon_{t}^{\epsilon_{0}}\left(\lambda, u^{\epsilon_{0}, k}\right)\right),
$$

where

$$
\Upsilon_{t}^{\epsilon_{0}}\left(\lambda, u^{\epsilon_{0}, k}\right):=\sup _{r \in[0, t]}\left(\left|\int_{0}^{r} f_{1, k}\left(s, u_{s}^{\epsilon_{0}, k}\right) d W_{s}^{1}\right|+\int_{0}^{r}(r-s)^{-\alpha-1}\left|\int_{s}^{r} f_{1, k}\left(\sigma, u_{\sigma}^{\epsilon_{0}, k}\right) d W_{\sigma}^{1}\right| d s\right) .
$$

Hence

$$
\begin{aligned}
\left\|u^{\epsilon_{0}, k}\right\|_{\alpha, \infty} & \leq e^{\lambda T}\left(\Psi_{T}^{\epsilon_{0}}\left(\lambda, u^{\epsilon_{0}, k}\right)+\Phi_{T}^{\epsilon_{0}}\left(\lambda, u^{\epsilon_{0}, k}\right)\right) \\
& \leq C_{\alpha, x, T} e^{C_{\alpha, x, T}\left(\Lambda_{\alpha}^{0, T}\left(B^{H, k}\right)\right)^{\frac{1}{1-\alpha}}}\left(1+\Lambda_{\alpha}^{0, T}\left(B^{H, k}\right)\right)^{\frac{1}{1-\alpha}}\left(1+\Upsilon_{T}^{\epsilon_{0}}\left(\lambda, u^{\epsilon_{0}, k}\right)\right) .
\end{aligned}
$$

By taking expectations on both sides of the above equation, we can get

$$
\begin{gathered}
\mathbb{E}\left\|u^{\epsilon_{0}, k}\right\|_{\alpha, \infty}^{p} \leq C_{\alpha, x, T}\left[\mathbb{E}\left[e^{3 p C_{\alpha, x, T}\left(\Lambda_{\alpha}^{0, T}\left(B^{H, k}\right)\right)^{\frac{1}{1-\alpha}}}\right]\left(1+\mathbb{E}\left[\Lambda_{\alpha}^{0, T}\left(B^{H, k}\right)\right]^{\frac{3 p}{1-\alpha}}\right)\right. \\
\left.\times\left(1+\mathbb{E}\left[\Upsilon_{T}^{\epsilon_{0}}\left(\lambda, u^{\epsilon_{0}, k}\right)\right]^{3 p}\right)\right]^{\frac{1}{3}}
\end{gathered}
$$

We need to prove that $\mathbb{E}\left[\Upsilon_{T}^{\epsilon_{0}}\left(\lambda, u^{\epsilon_{0}, k}\right)\right]^{p}$ is bounded for any $p \geq 1$. Applying the GarsiaRodemich-Rumsey inequality $(2.2)$ with $p=2 / \eta$ and $\theta=(1-\eta) / 2$ (where $\eta \in(0,1 / 2-\alpha)$ ), it deduce that

$$
\left|\int_{s}^{t} f_{1, k}\left(r, u_{r}^{\epsilon_{0}, k}\right) d W_{r}^{1}\right| \leq C_{\eta}|t-s|^{1 / 2-\eta} \zeta
$$

where

$$
\zeta=\left(\int_{0}^{T} \int_{0}^{T}|\sigma-r|^{-1 / \eta}\left|\int_{r}^{\sigma} f_{1, k}\left(z, u_{z}^{\epsilon_{0}, k}\right) d W_{z}^{1}\right|^{2 / \eta} d \sigma d r\right)^{\eta / 2}
$$


Then, taking expectations for $\zeta$, we can get

$$
\mathbb{E} \zeta^{p}=\int_{0}^{T} \int_{0}^{T}|\sigma-r|^{-p / 2} \mathbb{E}\left(\int_{r}^{\sigma}\left|f_{1, k}\left(z, u_{z}^{\epsilon_{0}, k}\right)\right|^{2} d z\right)^{p / 2} d \sigma d r \leq C_{T} .
$$

Fixed $\eta \in(0,1 / 2-\alpha)$, it follows that

$$
\begin{aligned}
\mathbb{E}\left[\Upsilon_{T}^{\epsilon_{0}}\left(\lambda, u^{\epsilon_{0}, k}\right)\right]^{p} & \leq C_{p} \int_{0}^{T} \mathbb{E}\left|f_{1, k}\left(r, u_{r}^{\epsilon_{0}, k}\right)\right|^{p} d r+C_{p} \mathbb{E} \zeta^{p}\left(\sup _{t \in[0, T]}\left|\int_{0}^{t}(t-s)^{-\eta-\alpha-1 / 2} d s\right|^{p}\right) \\
& \leq C_{\alpha, p, T}
\end{aligned}
$$

Thanks to (3.11), (3.13) and $\Lambda_{\alpha}^{0, T}\left(B^{H, k}\right)$ has moments of all orders [20], it yields (3.5).

Next, we estimate $\mathbb{E}\left\|v_{t}^{\epsilon_{0}, k}\right\|_{\alpha, \infty}^{2}$

$$
\begin{aligned}
\mathbb{E}\left\|v^{\epsilon_{0}, k}\right\|_{\alpha, \infty}^{p} \leq C_{p, y, \epsilon_{0}}\left(1+\mathbb{E}\left[\sup _{t \in[0, T]}\left|\int_{0}^{t} b_{2, k}\left(r, u_{r}^{\epsilon_{0}, k}, v_{r}^{\epsilon_{0}, k}\right) d r\right|\right]^{p}+\Gamma_{T}^{\epsilon_{0}}\left(b_{2, k}\right)\right. \\
\left.+\mathbb{E}\left[\sup _{t \in[0, T]}\left|\int_{0}^{t} f_{2}\left(r, v_{r}^{\epsilon_{0}, k}\right) d W_{r}^{2}\right|\right]^{p}+\Lambda_{T}^{\epsilon_{0}}\left(f_{2}\right)\right) \\
\leq C_{p, y, \epsilon_{0}, T}\left(1+\int_{0}^{T}\left(\mathbb{E}\left|u_{r}^{\epsilon_{0}, k}\right|^{p}+\mathbb{E}\left|v_{r}^{\epsilon_{0}, k}\right|^{p}\right) d r+\Gamma_{T}^{\epsilon_{0}}\left(b_{2, k}\right)+\Lambda_{T}^{\epsilon_{0}}\left(f_{2}\right)\right)(3 .
\end{aligned}
$$

where

$$
\Gamma_{T}^{\epsilon_{0}}\left(b_{2, k}\right):=\mathbb{E}\left(\sup _{t \in[0, T]} \int_{0}^{t}(t-s)^{-\alpha-1}\left|\int_{s}^{t} b_{2, k}\left(r, u_{r}^{\epsilon_{0}, k}, v_{r}^{\epsilon_{0}, k}\right) d r\right| d s\right)^{p}
$$

and

$$
\Lambda_{T}^{\epsilon_{0}}\left(f_{2}\right):=\mathbb{E}\left(\sup _{t \in[0, T]} \int_{0}^{t}(t-s)^{-\alpha-1}\left|\int_{s}^{t} f_{2}\left(r, v_{r}^{\epsilon_{0}, k}\right) d W_{r}^{2}\right| d s\right)^{p} .
$$

For $\Gamma_{T}^{\epsilon_{0}}\left(b_{2, k}\right)$, applying the Garsia-Rodemich-Rumsey inequality (2.2) with $p=2 / \varrho$ and $\theta=(1-\varrho) / 2($ where $\varrho \in(0,1 / 2-\alpha))$ again, we also can get

$$
\left|\int_{s}^{t} b_{2, k}\left(r, u_{r}^{\epsilon_{0}, k}, v_{r}^{\epsilon_{0}, k}\right) d r\right| \leq C_{\varrho}|t-s|^{1 / 2-\varrho} \varsigma
$$

where

$$
\varsigma=\left(\int_{0}^{T} \int_{0}^{T}|\sigma-r|^{-1 / \varrho}\left|\int_{r}^{\sigma} b_{2, k}\left(z, u_{z}^{\epsilon_{0}, k}, v_{z}^{\epsilon_{0}, k}\right) d z\right|^{2 / \varrho} d \sigma d r\right)^{\varrho / 2} .
$$

Then, taking expectations for $\varsigma$, we can get

$$
\begin{aligned}
\mathbb{E} \varsigma^{p} & \leq \int_{0}^{T} \int_{0}^{T}|\sigma-r|^{-1+p / 2} \int_{r}^{\sigma} \mathbb{E}\left|b_{2, k}\left(z, u_{z}^{\epsilon_{0}, k}, v_{z}^{\epsilon_{0}, k}\right)\right|^{p} d z d \sigma d r \\
& \leq \int_{0}^{T} \int_{0}^{T}|\sigma-r|^{-1+p / 2} d \sigma d r \int_{0}^{T} \mathbb{E}\left|b_{2, k}\left(z, u_{z}^{\epsilon_{0}, k}, v_{z}^{\epsilon_{0}, k}\right)\right|^{p} d z
\end{aligned}
$$




$$
\leq C_{p, T}\left(1+\int_{0}^{T} \mathbb{E}\left|u_{r}^{\epsilon_{0}, k}\right|^{p} d r+\int_{0}^{T} \mathbb{E}\left|v_{r}^{\epsilon_{0}, k}\right|^{p} d r\right)
$$

Hence

$$
\begin{aligned}
\Gamma_{T}^{\epsilon_{0}}\left(b_{2, k}\right) & \leq C \mathbb{E} \varsigma^{p}\left(\sup _{t \in[0, T]} \int_{0}^{t}(t-s)^{-\varrho-\alpha-1 / 2} d s\right)^{p} \\
& \leq C_{\alpha, p, T}\left(1+\int_{0}^{T} \mathbb{E}\left|u_{r}^{\epsilon_{0}, k}\right|^{p} d r+\int_{0}^{T} \mathbb{E}\left|v_{r}^{\epsilon_{0}, k}\right|^{p} d r\right)
\end{aligned}
$$

Moreover, use the same argument as (3.13) and (3.15), we also can obtain

$$
\Lambda_{T}^{\epsilon_{0}}\left(f_{2}\right) \leq C_{\alpha, p, T}\left(1+\int_{0}^{T} \mathbb{E}\left|v_{r}^{\epsilon_{0}, k}\right|^{p} d r\right) .
$$

Substituting (3.15) and (3.16) into (3.14), thanks to (3.5), we have

$$
\mathbb{E}\left\|v^{\epsilon_{0}, k}\right\|_{\alpha, \infty}^{p}=\mathbb{E}\left(\sup _{t \in[0, T]}\left\|v_{t}^{\epsilon_{0}, k}\right\|_{\alpha}\right)^{p} \leq C_{\alpha, p, \epsilon_{0}, x, y, T}\left(1+\int_{0}^{T} \mathbb{E}\left(\sup _{\sigma \in[0, r]}\left\|v_{\sigma}^{\epsilon_{0}, k}\right\|_{\alpha}\right)^{p} d r\right) .
$$

Then, by Gronwall inequality, we have (3.6). The proof is completed.

\subsection{The existence and uniqueness of solutions}

Now, we study the existence and uniqueness of solutions for original equation (1.1):

Proof of Theorem 2.4: In order to prove the existence of the solution for (1.1), fixed $\epsilon_{0} \in(0,1]$ and any $k \in \mathbb{N}$, we define the following stopping time

$$
\tau_{k}^{1}:=\inf \left\{t \geq 0:\left\|u_{t}^{\epsilon_{0}, k}\right\|_{\alpha}+\left\|v_{t}^{\epsilon_{0}, k}\right\|_{\alpha} \geq k\right\} \wedge \tau_{k}
$$

and we let

$$
\tau:=\sup _{k \in \mathbb{N}} \tau_{k}^{1}
$$

It is easy to know that the sequence of stopping times $\left\{\tau_{k}^{1}\right\}$ is non-decreasing and $\mathbb{P}(\tau=$ $+\infty)=1$. Indeed,

$$
\mathbb{P}(\tau<+\infty)=\lim _{T \rightarrow+\infty} \mathbb{P}(\tau \leq T),
$$

and for each $T>0$, thanks to Lemma 3.1 and Lemma 7.5 in [20], we can get

$$
\begin{aligned}
\mathbb{P}(\tau \leq T) & =\lim _{k \rightarrow+\infty} \mathbb{P}\left(\tau_{k}^{1} \leq T\right) \\
& =\lim _{k \rightarrow+\infty} \mathbb{P}\left(\sup _{t \in[0, T]}\left\|u_{t}^{\epsilon_{0}, k}\right\|_{\alpha}+\sup _{t \in[0, T]}\left\|v_{t}^{\epsilon_{0}, k}\right\|_{\alpha}+\sup _{t \in[0, T]} \Lambda_{\alpha}^{0, t}\left(B^{H}\right) \geq k\right) \\
& =\lim _{k \rightarrow+\infty} \frac{1}{k^{2}} \mathbb{E}\left(\sup _{t \in[0, T]}\left\|u_{t}^{\epsilon_{0}, k}\right\|_{\alpha}^{2}+\sup _{t \in[0, T]}\left\|v_{t}^{\epsilon_{0}, k}\right\|_{\alpha}^{2}+\frac{1}{\Gamma^{2}(1-\alpha)} \sup _{0<s<t<T}\left|\left(D_{t-}^{1-\alpha} B_{t-}^{H}\right)(s)\right|^{2}\right) \\
& =0 .
\end{aligned}
$$


Hence, $P(\tau<+\infty)=0$, that is, $P(\tau=+\infty)=1$. Further, for any $t \in[0, T]$ and $\omega \in$ $\{\tau=+\infty\}$, there exists $l \in \mathbb{N}$ such that $t \leq \tau_{l}^{1}(\omega)$. Then, we define

$$
u_{t}^{\epsilon_{0}}(\omega):=u_{t}^{\epsilon_{0}, l}(\omega) \quad \text { and } \quad v_{t}^{\epsilon_{0}}(\omega):=v_{t}^{\epsilon_{0}, l}(\omega) .
$$

This is a good definition, as for any $t \leq \tau_{k}^{1} \wedge \tau_{l}^{1}$, we have

$$
u_{t}^{\epsilon_{0}, k}=u_{t}^{\epsilon_{0}, l} \quad \text { and } \quad v_{t}^{\epsilon_{0}, k}=v_{t}^{\epsilon_{0}, l}, \quad \mathbb{P}-\text { a.s. }
$$

Actually, for any $k \geq l$ and $t \leq \tau_{k}^{1} \wedge \tau_{l}^{1}$, thanks to (3.3) and (3.4), we have

$$
\begin{aligned}
u_{t}^{\epsilon_{0}, k}-u_{t}^{\epsilon_{0}, l}= & \int_{0}^{t}\left[b_{1, k}\left(r, u_{r}^{\epsilon_{0}, k}, v_{r}^{\epsilon_{0}, k}\right)-b_{1, l}\left(r, u_{r}^{\epsilon_{0}, l}, v_{r}^{\epsilon_{0}, l}\right)\right] d r \\
& +\int_{0}^{t}\left[f_{1, k}\left(r, u_{r}^{\epsilon_{0}, k}\right)-f_{1, l}\left(r, u_{r}^{\epsilon_{0}, l}\right)\right] d W_{r}^{1} \\
& +\int_{0}^{t} g_{1, k}\left(r, u_{r}^{\epsilon_{0}, k}\right) d B_{r}^{H, k}-\int_{0}^{t} g_{1, l}\left(r, u_{r}^{\epsilon_{0}, l}\right) d B_{r}^{H, l} \\
= & \int_{0}^{t}\left[b_{1, k}\left(r, u_{r}^{\epsilon_{0}, k}, v_{r}^{\epsilon_{0}, k}\right)-b_{1, k}\left(r, u_{r}^{\epsilon_{0}, l}, v_{r}^{\epsilon_{0}, l}\right)\right] d r \\
& +\int_{0}^{t}\left[f_{1, k}\left(r, u_{r}^{\epsilon_{0}, k}\right)-f_{1, k}\left(r, u_{r}^{\epsilon_{0}, l}\right)\right] d W_{r}^{1} \\
& +\int_{0}^{t}\left[g_{1, k}\left(r, u_{r}^{\epsilon_{0}, k}\right)-g_{1, k}\left(r, u_{r}^{\epsilon_{0}, l}\right)\right] d B_{r}^{H, k} .
\end{aligned}
$$

Similarly, we also have

$$
\begin{aligned}
v_{t}^{\epsilon_{0}, k}-v_{t}^{\epsilon_{0}, l}= & \frac{1}{\epsilon_{0}} \int_{0}^{t}\left[b_{2, k}\left(r, u_{r}^{\epsilon_{0}, k}, v_{r}^{\epsilon_{0}, k}\right)-b_{2, k}\left(r, u_{r}^{\epsilon_{0}, l}, v_{r}^{\epsilon_{0}, l}\right)\right] d r \\
& +\frac{1}{\sqrt{\epsilon_{0}}} \int_{0}^{t}\left[f_{2}\left(r, u_{r}^{\epsilon_{0}, k}, v_{r}^{\epsilon_{0}, k}\right)-f_{2}\left(r, u_{r}^{\epsilon_{0}, l}, v_{r}^{\epsilon_{0}, l}\right)\right] d W_{r}^{2} .
\end{aligned}
$$

According to the paper [20] and [17], we know that the trajectories of $u_{t}^{\epsilon_{0}, k}$ and $u_{t}^{\epsilon_{0}, l}$ are $\eta$-Hölder continuous for all $\eta<1 / 2$. Now, let $\eta \in\left(\frac{\alpha}{\gamma}, \frac{1}{2}\right)$ and consider the set $\Omega_{N} \subset \Omega$ with $N \in \mathbb{N}$, such that

$$
\Omega_{N}:=\left\{\omega \in\{\tau=+\infty\}:\left\|u^{\epsilon_{0}, k}\right\|_{\eta} \leq N \text { and }\left\|u^{\epsilon_{0}, l}\right\|_{\eta} \leq N\right\} .
$$

It is clear that $\Omega_{N} \nearrow\{\tau=+\infty\}$. Then, by proceeding as Proposition 3.4, Proposition 3.6 and Proposition 3.9 in [17], we can get

$$
\begin{aligned}
& \mathbb{E}\left[\left\|u_{t}^{\epsilon_{0}, k}-u_{t}^{\epsilon_{0}, l}\right\|_{\alpha}^{2} \mathbf{1}_{\Omega_{N}}\right]+\mathbb{E}\left[\left\|v_{t}^{\epsilon_{0}, k}-v_{t}^{\epsilon_{0}, l}\right\|_{\alpha}^{2} \mathbf{1}_{\Omega_{N}}\right] \\
\leq & C_{\alpha, T} \mathbb{E}\left(\int_{0}^{t}(t-r)^{-\alpha}\left\|b_{1, k}\left(r, u_{r}^{\epsilon_{0}, k}, v_{r}^{\epsilon_{0}, k}\right)-b_{1, k}\left(r, u_{r}^{\epsilon_{0}, l}, v_{r}^{\epsilon_{0}, l}\right)\right\|_{\alpha} \mathbf{1}_{\Omega_{N}} d r\right)^{2} \\
+ & C_{\alpha, T} \int_{0}^{t}(t-r)^{-\frac{1}{2}-\alpha} \mathbb{E}\left[\left\|f_{1, k}\left(r, u_{r}^{\epsilon_{0}, k}\right)-f_{1, k}\left(r, u_{r}^{\epsilon_{0}, l}\right)\right\|_{\alpha}^{2} \mathbf{1}_{\Omega_{N}}\right] d r
\end{aligned}
$$




$$
\begin{aligned}
& +C_{\alpha, T} \mathbb{E}\left[\Lambda_{\alpha}^{0, t}\left(B^{H, k}\right) \int_{0}^{t}\left((t-r)^{-2 \alpha}+r^{-\alpha}\right)\left(1+\Delta u_{r}^{\epsilon_{0}, k}+\Delta u_{r}^{\epsilon_{0}, l}\right)\left\|u_{r}^{\epsilon_{0}, k}-u_{r}^{\epsilon_{0}, l}\right\|_{\alpha} \mathbf{1}_{\Omega_{N}} d r\right]^{2} \\
& +C_{\alpha, \epsilon_{0}, T} \mathbb{E}\left(\int_{0}^{t}(t-r)^{-\alpha}\left\|b_{2, k}\left(r, u_{r}^{\epsilon_{0}, k}, v_{r}^{\epsilon_{0}, k}\right)-b_{2, k}\left(r, u_{r}^{\epsilon_{0}, l}, v_{r}^{\epsilon_{0}, l}\right)\right\|_{\alpha} \mathbf{1}_{\Omega_{N}} d r\right)^{2} \\
& +C_{\alpha, \epsilon_{0}, T} \int_{0}^{t}(t-r)^{-\frac{1}{2}-\alpha} \mathbb{E}\left[\left\|f_{2}\left(r, u_{r}^{\epsilon_{0}, k}, v_{r}^{\epsilon_{0}, k}\right)-f_{2}\left(r, u_{r}^{\epsilon_{0}, l}, v_{r}^{\epsilon_{0}, l}\right)\right\|_{\alpha}^{2} \mathbf{1}_{\Omega_{N}}\right] d r \\
\leq & C_{\alpha, \epsilon_{0}, T} \int_{0}^{t}\left((t-r)^{-2 \alpha}+(t-r)^{-\frac{1}{2}-\alpha}\right)\left(\mathbb{E}\left[\left\|u_{r}^{\epsilon_{0}, k}-u_{r}^{\epsilon_{0}, l}\right\|_{\alpha}^{2} \mathbf{1}_{\Omega_{N}}\right]+\mathbb{E}\left[\left\|v_{r}^{\epsilon_{0}, k}-v_{r}^{\epsilon_{0}, l}\right\|_{\alpha}^{2} \mathbf{1}_{\Omega_{N}}\right]\right) d r \\
& +C_{\alpha, k, k, T} \int_{0}^{t}\left((t-r)^{-2 \alpha}+r^{-\alpha}\right) \mathbb{E}\left[\left\|u_{r}^{\epsilon_{0}, k}-u_{r}^{\epsilon_{0}, l}\right\|_{\alpha}^{2} \mathbf{1}_{\Omega_{N}}\right] d r \\
\leq & C_{\alpha, \epsilon_{0}, k, k, T} \int_{0}^{t}(t-r)^{-\frac{1}{2}-\alpha} r^{-\frac{1}{2}-\alpha}\left(\mathbb{E}\left[\left\|u_{r}^{\epsilon_{0}, k}-u_{r}^{\epsilon_{0}, l}\right\|_{\alpha}^{2} \mathbf{1}_{\Omega_{N}}\right]+\mathbb{E}\left[\left\|v_{r}^{\epsilon_{0}, k}-v_{r}^{\epsilon_{0}, l}\right\|_{\alpha}^{2} \mathbf{1}_{\Omega_{N}}\right]\right) d r
\end{aligned}
$$

where the last estimate is because $\Lambda_{\alpha}^{0, t}\left(B^{H, k}\right) \leq k$ and

$$
\begin{aligned}
1+\Delta u_{r}^{\epsilon_{0}, k}+\Delta u_{r}^{\epsilon_{0}, l} & =1+\int_{0}^{r} \frac{\left|u_{r}^{\epsilon_{0}, k}-u_{s}^{\epsilon_{0}, k}\right|^{\gamma}}{(r-s)^{\alpha+1}} d s+\int_{0}^{r} \frac{\left|u_{r}^{\epsilon_{0}, l}-u_{s}^{\epsilon_{0}, l}\right|^{\gamma}}{(r-s)^{\alpha+1}} d s \\
& \leq 1+\left(\left\|u^{\epsilon_{0}, k}\right\|_{\eta}^{\gamma}+\left\|u^{\epsilon_{0}, l}\right\|_{\eta}^{\gamma}\right) \int_{0}^{r}(r-s)^{\eta \gamma-\alpha-1} d s \leq C_{N},
\end{aligned}
$$

as $t \leq \tau_{k}^{1} \wedge \tau_{l}^{1}$ and $\Omega_{N} \subset\{\tau=+\infty\}$. Therefore, for any $k \in \mathbb{N}$, by the Gronwall-type lemma (Lemma 7.6 in [20]), we deduce that

$$
\mathbb{E}\left[\left\|u_{t}^{\epsilon_{0}, k}-u_{t}^{\epsilon_{0}, l}\right\|_{\alpha}^{2} \mathbf{1}_{\Omega_{N}}\right]+\mathbb{E}\left[\left\|v_{t}^{\epsilon_{0}, k}-v_{t}^{\epsilon_{0}, l}\right\|_{\alpha}^{2} \mathbf{1}_{\Omega_{N}}\right]=0, \quad t \leq \tau_{k} \wedge \tau_{l} .
$$

Then, let $N \rightarrow+\infty$, as $\Omega_{N} \nearrow\{\tau=+\infty\}$, we can get (3.21).

Recalling that if $\omega \in\{\tau=+\infty\}$ and $t \leq \tau_{l}$, we denote $u_{t}^{\epsilon_{0}}$ is equal to $u_{t}^{\epsilon_{0}, l}$ and $v_{t}^{\epsilon_{0}}$ is equal to $v_{t}^{\epsilon_{0}, l}$, thanks to (3.3) and (3.4), it follows that

$$
\left\{\begin{array}{l}
u_{t}^{\epsilon_{0}}=x+\int_{0}^{t} b_{1}\left(r, u_{r}^{\epsilon_{0}}, v_{r}^{\epsilon_{0}}\right) d r+\int_{0}^{t} f_{1}\left(r, u_{r}^{\epsilon_{0}}\right) d W_{r}^{1}+\int_{0}^{t} g_{1}\left(r, u_{r}^{\epsilon_{0}}\right) d B_{r}^{H}, \\
v_{t}^{\epsilon_{0}}=y+\frac{1}{\epsilon_{0}} \int_{0}^{t} b_{2}\left(r, u_{r}^{\epsilon_{0}}, v_{r}^{\epsilon_{0}}\right) d r+\frac{1}{\sqrt{\epsilon_{0}}} \int_{0}^{t} f_{2}\left(r, u_{r}^{\epsilon_{0}}, v_{r}^{\epsilon_{0}}\right) d W_{r}^{2}
\end{array}\right.
$$

$\mathbb{P}$-a.s., that is, for any fixed $\epsilon_{0} \in(0,1],\left(u_{t}^{\epsilon_{0}}, v_{t}^{\epsilon_{0}}\right)$ is a solution of equation (1.1).

Finally, denote another solution of equation (1.1) is $\left(u_{t}^{\epsilon_{0}, *}, v_{t}^{\epsilon_{0}, *}\right)$, with the same argument as (3.21), we can also get that

$$
u_{t}^{\epsilon_{0}}=u_{t}^{\epsilon_{0}, *} \quad \text { and } \quad v_{t}^{\epsilon_{0}}=v_{t}^{\epsilon_{0}, *}, \quad \mathbb{P}-\text { a.s. }
$$

Thus, we prove the uniqueness of the solution of equation (1.1). This proof is completed.

\section{Proof of Theorem 2.5}

In this section, we prove the main Theorem 2.5, i.e. the slow process $u_{t}^{\epsilon}$ converges to the averaged process $\bar{u}_{t}$ in the mean square sense, as $\epsilon \rightarrow 0$. Firstly, we need to define 
the averaged equation and give some properties of the averaged coefficient. Secondly, we construct an auxiliary process $\hat{v}_{t}^{\epsilon}$ by the technique of time discretization and give some estimates about it on the basis of some a-priori estimates for the solution $\left(u_{t}^{\epsilon}, v_{t}^{\epsilon}\right)$ of original equation (1.1) are given. Finally, we construct the stopping time and obtain appropriate control of $u_{t}^{\epsilon}-\bar{u}_{t}$ before and after the stopping time respectively.

\subsection{The averaged equation}

To define the averaged equation, we first consider the equation (1.3) associated to the fast equation.

Under the assumptions (A1)-(A5), it is easy to prove that the equation (1.3) has a unique strong solution $v_{t}^{s, x, y}$, which is a time homogeneous Markov process. Moreover, use the same argument as [15], there exists some constant $\beta_{p}^{*}>0$ such that the following estimates hold and we will not give a detailed proof here:

$$
\mathbb{E}\left|v_{t}^{s, x, y}\right|^{p} \leq C_{p, T}\left(1+|x|^{p}\right)+e^{-\beta_{p}^{*} t}|y|^{p},
$$

and

$$
\mathbb{E}\left|v_{t}^{s, x, y_{1}}-v_{t}^{s, x, y_{2}}\right|^{2} \leq e^{-\beta_{1} t}\left|y_{1}-y_{2}\right|^{2}
$$

and

$$
\mathbb{E}\left|v_{t}^{s_{1}, x_{1}, y}-v_{t}^{s_{2}, x_{2}, y}\right|^{2} \leq C_{T}\left(\left|s_{1}-s_{2}\right|^{2 \iota}+\left|x_{1}-x_{2}\right|^{2}\right)\left(1+\left|x_{1}\right|^{2 \alpha_{1}}+\left|x_{2}\right|^{2 \alpha_{1} \vee 2 \alpha_{2}}+|y|^{2 \alpha_{2}}\right) .
$$

Let $\left\{P_{t}^{s, x}\right\}_{t \geq 0}$ be the transition semigroup of $\left\{v_{t}^{s, x, y}\right\}_{t \geq 0}$, that is

$$
P_{t}^{s, x} \varphi(y):=\mathbb{E} \varphi\left(v_{t}^{s, x, y}\right), \quad s>0, y \in \mathbb{R}^{m}
$$

where $\varphi: \mathbb{R}^{m} \rightarrow \mathbb{R}$ is a bounded measurable function.

Then, we can establish the following crucial lemma:

Lemma 4.1. Assume that the conditions (A1)-(A5) hold. Then, for any fixed $s>0$ and $x \in \mathbb{R}^{n}$, there exists a unique invariant measure $\mu^{s, x}$ for the equation (1.3), and

$$
\int_{\mathbb{R}^{m}}|z|^{p} \mu^{s, x}(d z) \leq C_{p, T}\left(1+|x|^{p}\right) .
$$

Moreover, for any $t>0$ and $y \in \mathbb{R}^{m}$, we obtain

$$
\left|\mathbb{E} b_{1}\left(s, x, v_{t}^{s, x, y}\right)-\int_{\mathbb{R}^{m}} b_{1}(s, x, z) \mu^{s, x}(d z)\right| \leq C_{T} e^{-\frac{\beta_{1}}{2} t}\left(1+|x|^{2\left(\theta_{2} \vee \theta_{3} \vee 1\right)}+|y|^{2\left(\theta_{3} \vee 1\right)}\right) .
$$

Proof: The detailed proof will be given in the Appendix.

Further, by the unique invariant measure $\mu^{s, x}$, the averaged equation can be defined as (1.5). Moreover, we can give some properties of the averaged coefficient $\bar{b}_{1}$, where the detailed proof of Lemma 4.2 can be found in the Appendix. 
Lemma 4.2. Assume that the conditions (A1)-(A5) hold. Then, for any $t \geq 0$ and $x \in \mathbb{R}^{n}$, we have

$$
\left|\bar{b}_{1}(t, x)\right| \leq C(1+|x|) .
$$

Moreover, for any $s_{1}, s_{2} \in[0, T], R \in \mathbb{R}$ and $x_{i} \in \mathbb{R}^{n}$ with $\left|x_{i}\right| \leq R$, we have

$$
\left|\bar{b}_{1}\left(s_{1}, x_{1}\right)-\bar{b}_{1}\left(s_{2}, x_{2}\right)\right| \leq C_{R, T}\left(\left|s_{1}-s_{2}\right|^{\iota}+\left|s_{1}-s_{2}\right|^{\kappa}+\left|x_{1}-x_{2}\right|\right) .
$$

Thanks to the assumptions and Lemma 4.2, by proceeding as Theorem 2.4 and Lemma 3.1, it is easy to get that there exists a unique strong solution $\bar{u}_{t}$ to equation (1.5) and we have

$$
\mathbb{E}\|\bar{u}\|_{\alpha, \infty}^{p} \leq C_{\alpha, p, x, T}, \quad p \geq 1 .
$$

\subsection{Some a-priori estimates}

To prove the Theorem 2.5, some a-priori estimates for the solution $\left(u_{t}^{\epsilon}, v_{t}^{\epsilon}\right)$ of original equation (1.1) need to be given at first.

Lemma 4.3. Assume that the conditions (A1)-(A5) hold. Then, for any $\epsilon \in(0,1], \alpha \in$ $\left(1-H, \frac{1}{2} \wedge \beta \wedge \frac{\gamma}{2}\right), p \geq 1$ and $t \in[0, T]$, we have

$$
\mathbb{E}\left\|u^{\epsilon}\right\|_{\alpha, \infty}^{p} \leq C_{\alpha, p, x, T} \quad \text { and } \quad \mathbb{E}\left|v_{t}^{\epsilon}\right|^{2} \leq C_{\alpha, x, y, T}
$$

Moreover, for any $h \in(0,1]$, it yields

$$
\mathbb{E}\left|u_{t+h}^{\epsilon}-u_{t}^{\epsilon}\right|^{2} \leq C_{\alpha, x, y, T} h
$$

Proof: According to the assumptions and use the same argument as Lemma 3.1 and [15, Lemma 3.1], it is easy to get that equation (4.10) hold. Moreover, using the Itô isometry for Brownian motion term and by proceeding as Lemma 4.2 in [22], we also can establish the equation (4.11). Here, we omit the detailed proof.

Then, inspired by Khasminskii's idea in [3], for any $\epsilon>0$, the interval $[0, T]$ is divided into subintervals of size $\delta_{\epsilon}>0$, where $\delta_{\epsilon}$ is a fixed number depending on $\epsilon$. Now, we construct a process $\hat{v}_{t}^{\epsilon}$ with initial value $\hat{v}_{0}^{\epsilon}=v_{0}^{\epsilon}=y$, and for $t \in\left[k \delta_{\epsilon}, \min \left\{(k+1) \delta_{\epsilon}, T\right\}\right]$, we have

$$
\hat{v}_{t}^{\epsilon}=\hat{v}_{k \delta_{\epsilon}}^{\epsilon}+\frac{1}{\epsilon} \int_{k \delta_{\epsilon}}^{t} b_{2}\left(k \delta_{\epsilon}, u_{k \delta_{\epsilon}}^{\epsilon}, \hat{v}_{r}^{\epsilon}\right) d r+\frac{1}{\sqrt{\epsilon}} \int_{k \delta_{\epsilon}}^{t} f_{2}\left(k \delta_{\epsilon}, u_{k \delta_{\epsilon}}^{\epsilon}, \hat{v}_{r}^{\epsilon}\right) d W_{r}^{2},
$$

i.e.,

$$
\left.\hat{v}_{t}^{\epsilon}=y+\frac{1}{\epsilon} \int_{0}^{t} b_{2}\left(r\left(\delta_{\epsilon}\right), u_{r\left(\delta_{\epsilon}\right)}^{\epsilon}\right) \hat{v}_{r}^{\epsilon}\right) d r+\frac{1}{\sqrt{\epsilon}} \int_{0}^{t} f_{2}\left(r\left(\delta_{\epsilon}\right), u_{r\left(\delta_{\epsilon}\right)}^{\epsilon}, \hat{v}_{r}^{\epsilon}\right) d W_{r}^{2},
$$

where $r\left(\delta_{\epsilon}\right)=\left\lfloor r / \delta_{\epsilon}\right\rfloor \delta_{\epsilon}$ is the nearest breakpoint preceding $r$. By the construction of $\hat{v}_{t}^{\epsilon}$, we have an estimate analogous to Lemma 4.3 hold, i.e., for any $t \in[0, T]$, we have

$$
\mathbb{E}\left|\hat{v}_{t}^{\epsilon}\right|^{2} \leq C_{\alpha, x, y, T}
$$

Moreover, thanks to the assumptions and Lemma 4.3, by proceeding as Lemma 3.4 in [15], it is easy to get that

$$
\mathbb{E}\left|v_{t}^{\epsilon}-\hat{v}_{t}^{\epsilon}\right|^{2} \leq C_{\alpha, x, y, T} \delta_{\epsilon}^{2 \iota \wedge 1}
$$




\subsection{The proof of Theorem 2.5}

Now, we construct the following stopping time $\tau_{R}^{\epsilon}$ for each $R \in \mathbb{R}$ :

$$
\tau_{R}^{\epsilon}:=\inf \left\{t \geq 0:\left\|u_{t}^{\epsilon}\right\|_{\alpha}+\left\|\bar{u}_{t}\right\|_{\alpha}+\Lambda_{\alpha}^{0, t}\left(B^{H}\right) \geq R\right\} .
$$

Moreover, due to the trajectories of $u_{t}^{\epsilon}$ and $\bar{u}_{t}$ are $\eta$-Hölder continuous for all $\eta<1 / 2$. As the proof in Theorem 2.4, let $\eta \in\left(\frac{\alpha}{\gamma}, \frac{1}{2}\right)$ and consider the following set $\Omega_{M} \subset \Omega$ with $M \in \mathbb{N}$, such that

$$
\Omega_{M}:=\left\{\omega \in \Omega:\left\|u^{\epsilon}\right\|_{\eta} \leq M \text { and }\|\bar{u}\|_{\eta} \leq M\right\} .
$$

It is clear that $\Omega_{M} \nearrow \Omega$.

First, we estimate the error of $u_{t}^{\epsilon}-\bar{u}_{t}$ before a stopping time:

Lemma 4.4. Assume that the conditions (A1)-(A5) hold. Then, for any $\alpha \in\left(1-H, \frac{1}{2} \wedge \beta\right.$ $\left.\wedge \frac{\gamma}{2}\right)$, we have

$$
\sup _{t \in[0, T]} \mathbb{E}\left[\left\|u_{t}^{\epsilon}-\bar{u}_{t}\right\|_{\alpha}^{2} \mathbf{1}_{\Omega_{M} \cap\left\{T \leq \tau_{R}^{\epsilon}\right\}}\right] \leq C_{\alpha, x, y, R, M, T}\left(\delta_{\epsilon}^{2 \kappa \wedge 2 \iota \wedge(1 / 2-\alpha)}+\epsilon / \delta_{\epsilon}\right) .
$$

Proof: It is easy to know that

$$
\begin{aligned}
\mathbb{E}\left[\left\|u_{t}^{\epsilon}-\bar{u}_{t}\right\|_{\alpha}^{2} \mathbf{1}_{\Omega_{M} \cap\left\{T \leq \tau_{R}^{\epsilon}\right\}}\right] \leq & 3 \mathbb{E}\left[\left\|\int_{0}^{t}\left(b_{1}\left(r, u_{r}^{\epsilon}, v_{r}^{\epsilon}\right)-\bar{b}_{1}\left(r, \bar{u}_{r}\right)\right) d r\right\|_{\alpha}^{2} \mathbf{1}_{\Omega_{M} \cap\left\{T \leq \tau_{R}^{\epsilon}\right\}}\right] \\
& +3 \mathbb{E}\left[\left\|\int_{0}^{t}\left(f_{1}\left(r, u_{r}^{\epsilon}\right)-f_{1}\left(r, \bar{u}_{r}\right)\right) d W_{r}^{1}\right\|_{\alpha}^{2} \mathbf{1}_{\Omega_{M} \cap\left\{T \leq \tau_{R}^{\epsilon}\right\}}\right] \\
& +3 \mathbb{E}\left[\left\|\int_{0}^{t}\left(g_{1}\left(r, u_{r}^{\epsilon}\right)-g_{1}\left(r, \bar{u}_{r}\right)\right) d B_{r}^{H}\right\|_{\alpha}^{2} \mathbf{1}_{\Omega_{M} \cap\left\{T \leq \tau_{R}^{\epsilon}\right\}}\right] \\
:= & 3 \mathcal{I}_{t}^{1}+3 \mathcal{I}_{t}^{2}+3 \mathcal{I}_{t}^{3} .
\end{aligned}
$$

For $\mathcal{I}_{t}^{2}$ and $\mathcal{I}_{t}^{3}$, if $\omega \in \Omega_{n}$ and $\eta \in\left(\frac{\alpha}{\gamma}, \frac{1}{2}\right)$, by proceeding as (3.22), use the conclusions of Proposition 3.6 and Proposition 3.9 in [17], it is easy to get that

$$
\mathcal{I}_{t}^{2}+\mathcal{I}_{t}^{3} \leq C_{\alpha, R, M, T} \int_{0}^{t}(t-r)^{-\frac{1}{2}-\alpha} r^{-\frac{1}{2}-\alpha} \mathbb{E}\left[\left\|u_{r}^{\epsilon}-\bar{u}_{r}\right\|_{\alpha}^{2} \mathbf{1}_{\Omega_{M} \cap\left\{T \leq \tau_{R}^{\epsilon}\right\}}\right] d r .
$$

For $\mathcal{I}_{t}^{1}$, we have

$$
\begin{aligned}
\mathcal{I}_{t}^{1} \leq & 4 \mathbb{E}\left[\left\|\int_{0}^{t} b_{1}\left(r, u_{r}^{\epsilon}, v_{r}^{\epsilon}\right)-b_{1}\left(r\left(\delta_{\epsilon}\right), u_{r\left(\delta_{\epsilon}\right)}^{\epsilon}, \hat{v}_{r}^{\epsilon}\right) d r\right\|_{\alpha}^{2} \mathbf{1}_{\Omega_{M} \cap\left\{T \leq \tau_{R}^{\epsilon}\right\}}\right] \\
& +4 \mathbb{E}\left[\left\|\int_{0}^{t} b_{1}\left(r\left(\delta_{\epsilon}\right), u_{r\left(\delta_{\epsilon}\right)}^{\epsilon}, \hat{v}_{r}^{\epsilon}\right)-\bar{b}_{1}\left(r\left(\delta_{\epsilon}\right), u_{r\left(\delta_{\epsilon}\right)}^{\epsilon}\right) d r\right\|_{\alpha}^{2} \mathbf{1}_{\Omega_{M} \cap\left\{T \leq \tau_{R}^{\epsilon}\right\}}\right] \\
& +4 \mathbb{E}\left[\left\|\int_{0}^{t} \bar{b}_{1}\left(r\left(\delta_{\epsilon}\right), u_{r\left(\delta_{\epsilon}\right)}^{\epsilon}\right)-\bar{b}_{1}\left(r, u_{r}^{\epsilon}\right) d r\right\|_{\alpha}^{2} \mathbf{1}_{\Omega_{M} \cap\left\{T \leq \tau_{R}^{\epsilon}\right\}}\right] \\
& +4 \mathbb{E}\left[\left\|\int_{0}^{t} \bar{b}_{1}\left(r, u_{r}^{\epsilon}\right)-\bar{b}_{1}\left(r, \bar{u}_{r}\right) d r\right\|_{\alpha}^{2} \mathbf{1}_{\Omega_{M} \cap\left\{T \leq \tau_{R}^{\epsilon}\right\}}\right]
\end{aligned}
$$




$$
:=4 \mathcal{J}_{t}^{1}+4 \mathcal{J}_{t}^{2}+4 \mathcal{J}_{t}^{3}+4 \mathcal{J}_{t}^{4}
$$

Thanks to Lemma 4.3 and equation (4.15), we can get

$$
\begin{aligned}
& \mathbb{E}\left[\left|\int_{s}^{t} b_{1}\left(r, u_{r}^{\epsilon}, v_{r}^{\epsilon}\right)-b_{1}\left(r\left(\delta_{\epsilon}\right), u_{r\left(\delta_{\epsilon}\right)}^{\epsilon}, \hat{v}_{r}^{\epsilon}\right) d r\right|^{2} \mathbf{1}_{\Omega_{M} \cap\left\{T \leq \tau_{R}^{\epsilon}\right\}}\right] \\
\leq & \mathbb{E}\left[\left|\int_{s}^{t} b_{1}\left(r, u_{r}^{\epsilon}, v_{r}^{\epsilon}\right)-b_{1}\left(r\left(\delta_{\epsilon}\right), u_{r}^{\epsilon}, v_{r}^{\epsilon}\right) d r\right|^{2} \mathbf{1}_{\Omega_{M} \cap\left\{T \leq \tau_{R}^{\epsilon}\right\}}\right] \\
& +\mathbb{E}\left[\left|\int_{s}^{t} b_{1}\left(r\left(\delta_{\epsilon}\right), u_{r}^{\epsilon}, v_{r}^{\epsilon}\right)-b_{1}\left(r\left(\delta_{\epsilon}\right), u_{r\left(\delta_{\epsilon}\right)}^{\epsilon}, v_{r}^{\epsilon}\right) d r\right|^{2} \mathbf{1}_{\left.\Omega_{M} \cap\left\{T \leq \tau_{R}^{\epsilon}\right\}\right]}\right] \\
& +\mathbb{E}\left[\left|\int_{s}^{t} b_{1}\left(r\left(\delta_{\epsilon}\right), u_{r\left(\delta_{\epsilon}\right)}^{\epsilon}, v_{r}^{\epsilon}\right)-b_{1}\left(r\left(\delta_{\epsilon}\right), u_{r\left(\delta_{\epsilon}\right)}^{\epsilon}, \hat{v}_{r}^{\epsilon}\right) d r\right|^{2} \mathbf{1}_{\left.\Omega_{M} \cap\left\{T \leq \tau_{R}^{\epsilon}\right\}\right]}\right] \\
\leq & C_{T} \int_{s}^{t}\left|r-r\left(\delta_{\epsilon}\right)\right|^{2 \kappa} \mathbb{E}\left[\left(1+\left|u_{r}^{\epsilon}\right|^{2 \theta_{2}}+\left|v_{r}^{\epsilon}\right|^{2 \theta_{3}}\right) \mathbf{1}_{\Omega_{M} \cap\left\{T \leq \tau_{R}^{\epsilon}\right\}}\right] d r \\
& +C_{R, T} \int_{s}^{t} \mathbb{E}\left[\left|u_{r}^{\epsilon}-u_{r\left(\delta_{\epsilon}\right)}^{\epsilon}\right|^{2} \mathbf{1}_{\Omega_{M} \cap\left\{T \leq \tau_{R}^{\epsilon}\right\}}\right] d r \int_{s}^{t} \mathbb{E}\left[\left(1+\left|v_{r}^{\epsilon}\right|^{2 \theta_{1}}\right) \mathbf{1}_{\left.\Omega_{M} \cap\left\{T \leq \tau_{R}^{\epsilon}\right\}\right] d r}\right. \\
& +C_{T} \int_{s}^{t} \mathbb{E}\left[\left|v_{r}^{\epsilon}-\hat{v}_{r}^{\epsilon}\right|^{2} \mathbf{1}_{\Omega_{M} \cap\left\{T \leq \tau_{R}^{\epsilon}\right\}}\right] d r \int_{s}^{t} \mathbb{E}\left[\left(1+\left|u_{r}^{\epsilon}\right|^{2 \theta_{2}}+\left|v_{r}^{\epsilon}\right|^{2 \theta_{3}}+\left|\hat{v}_{r}^{\epsilon}\right|^{2 \theta_{3}}\right) \mathbf{1}_{\Omega_{M} \cap\left\{T \leq \tau_{R}^{\epsilon}\right\}}\right] d r \\
\leq & C_{\alpha, x, y, R, T}\left(\delta_{\epsilon}^{2 \kappa}+\delta_{\epsilon}+\delta_{\epsilon}^{2 \wedge 1}\right)(t-s) .
\end{aligned}
$$

Hence

$$
\begin{aligned}
\mathcal{J}_{t}^{1} \leq & 2 \mathbb{E}\left[\left|\int_{0}^{t} b_{1}\left(r, u_{r}^{\epsilon}, v_{r}^{\epsilon}\right)-b_{1}\left(r\left(\delta_{\epsilon}\right), u_{r\left(\delta_{\epsilon}\right)}^{\epsilon}, \hat{v}_{r}^{\epsilon}\right) d r\right|^{2} \mathbf{1}_{\Omega_{M} \cap\left\{T \leq \tau_{R}^{\epsilon}\right\}}\right] \\
& +2 \mathbb{E}\left[\left|\int_{0}^{t}(t-r)^{-\alpha-1}\right| \int_{r}^{t} b_{1}\left(s, u_{s}^{\epsilon}, v_{s}^{\epsilon}\right)-b_{1}\left(s\left(\delta_{\epsilon}\right), u_{s\left(\delta_{\epsilon}\right)}^{\epsilon}, \hat{v}_{s}^{\epsilon}\right) d s|d r|^{2} \mathbf{1}_{\Omega_{M} \cap\left\{T \leq \tau_{R}^{\epsilon}\right\}}\right] \\
\leq & C_{\alpha, x, y, R, T}\left(\delta_{\epsilon}^{2 \kappa}+\delta_{\epsilon}+\delta_{\epsilon}^{2 \iota \wedge 1}\right)+C \int_{0}^{t}(t-r)^{-\alpha-\frac{1}{2}} d r \\
& \quad \times \int_{0}^{t}(t-r)^{-\alpha-\frac{3}{2}} \mathbb{E}\left[\left|\int_{r}^{t} b_{1}\left(s, u_{s}^{\epsilon}, v_{s}^{\epsilon}\right)-b_{1}\left(s\left(\delta_{\epsilon}\right), u_{s\left(\delta_{\epsilon}\right)}^{\epsilon}, \hat{v}_{s}^{\epsilon}\right) d s\right|^{2} \mathbf{1}_{\Omega_{M} \cap\left\{T \leq \tau_{R}^{\epsilon}\right\}}\right] d r \\
\leq & C_{\alpha, x, y, R, T}\left(\delta_{\epsilon}^{2 \kappa}+\delta_{\epsilon}+\delta_{\epsilon}^{2 \iota \wedge 1}\right) \leq C_{\alpha, x, y, R, T} \delta_{\epsilon}^{2 \kappa \wedge 2 \iota \wedge 1} .
\end{aligned}
$$

Then, due to (4.8) and Proposition 3.4 in [17], by proceeding as (4.21), it is easy to get that

$$
\mathcal{J}_{t}^{3}+\mathcal{J}_{t}^{4} \leq C_{\alpha, R, x, y, T} \delta_{\epsilon}^{2 \kappa \wedge 2 \iota \wedge 1}+C_{\alpha, R, T} \int_{0}^{t}(t-r)^{-2 \alpha} \mathbb{E}\left[\left\|u_{r}^{\epsilon}-\bar{u}_{r}\right\|_{\alpha}^{2} \mathbf{1}_{\Omega_{M} \cap\left\{T \leq \tau_{R}^{\epsilon}\right\}}\right] d r .
$$

In order to prove the approximation result of the equation (4.16), we must estimate $\mathcal{J}_{t}^{2}$. Therefore, we establish the following crucial lemma:

Lemma 4.5. Assume that the conditions (A1)-(A5) hold. Then, for any $s, \Lambda>0, x \in \mathbb{R}^{n}$ and $y \in \mathbb{R}^{m}$, we have

$$
\mathbb{E}\left|\frac{1}{\Lambda} \int_{0}^{\Lambda} b_{1}\left(s, x, v_{r}^{s, x, y}\right) d r-\bar{b}_{1}(s, x)\right|^{2} \leq \frac{C}{\Lambda}\left(1+|x|^{4\left(\theta_{2} \vee \theta_{3} \vee 1\right)}+|y|^{4\left(\theta_{3} \vee 1\right)}\right) .
$$


Proof: Using the Markov property of $\left\{v_{t}^{s, x, y}\right\}_{t \geq 0}$, we have

$$
\begin{aligned}
& \mathbb{E}\left|\frac{1}{\Lambda} \int_{0}^{\Lambda} b_{1}\left(s, x, v_{r}^{s, x, y}\right) d r-\bar{b}_{1}(s, x)\right|^{2} \\
= & \frac{2}{\Lambda^{2}} \int_{0}^{\Lambda} \int_{r}^{\Lambda} \mathbb{E}\left[\left(b_{1}\left(s, x, v_{r}^{s, x, y}\right)-\bar{b}_{1}(s, x)\right)\left(b_{1}\left(s, x, v_{\sigma}^{s, x, y}\right)-\bar{b}_{1}(s, x)\right)\right] d \sigma d r \\
= & \frac{2}{\Lambda^{2}} \int_{0}^{\Lambda} \int_{r}^{\Lambda} \mathbb{E}\left[\left(b_{1}\left(s, x, v_{r}^{s, x, y}\right)-\bar{b}_{1}(s, x)\right) P_{\sigma-r}^{s, x}\left(b_{1}\left(s, x, v_{r}^{s, x, y}\right)-\bar{b}_{1}(s, x)\right)\right] d \sigma d r \\
\leq & \frac{2}{\Lambda^{2}} \int_{0}^{\Lambda} \int_{r}^{\Lambda}\left[\mathbb{E}\left|b_{1}\left(s, x, v_{r}^{s, x, y}\right)-\bar{b}_{1}(s, x)\right|^{2} \mathbb{E}\left|P_{\sigma-r}^{s, x}\left(b_{1}\left(s, x, v_{r}^{s, x, y}\right)-\bar{b}_{1}(s, x)\right)\right|^{2}\right]^{\frac{1}{2}} d \sigma d r .
\end{aligned}
$$

By assumption (A1) and thanks to (4.1), (4.7), we can get

$$
\begin{aligned}
\mathbb{E}\left|b_{1}\left(s, x, v_{r}^{s, x, y}\right)-\bar{b}_{1}(s, x)\right|^{2} & \leq 2 \mathbb{E}\left|b_{1}\left(s, x, v_{r}^{s, x, y}\right)\right|^{2}+2 \mathbb{E}\left|\bar{b}_{1}(s, x)\right|^{2} \\
& \leq C\left(1+|x|^{2}+\mathbb{E}\left|v_{r}^{s, x, y}\right|^{2}\right) \\
& \leq C\left(1+|x|^{2}\right)+e^{-\beta_{2}^{*} t}|y|^{2}
\end{aligned}
$$

Then, according to the equation (4.6) and (4.1), we obtain

$$
\begin{aligned}
\mathbb{E}\left|P_{\sigma-r}^{s, x}\left(b_{1}\left(s, x, v_{r}^{s, x, y}\right) d r-\bar{b}_{1}(s, x)\right)\right|^{2} & =\mathbb{E}\left|\mathbb{E} b_{1}\left(s, x, v_{\sigma-r}^{s, x, v_{r}^{s, x, y}}\right)-\int_{\mathbb{R}^{m}} b_{1}(s, x, z) \mu^{s, x}(d z)\right|^{2} \\
& \leq\left. C_{T} e^{-\frac{\beta_{1}}{2}(\sigma-r)} \mathbb{E}|1+| x\right|^{2\left(\theta_{2} \vee \theta_{3} \vee 1\right)}+\left.\mathbb{E}\left|v_{r}^{s, x, y}\right|^{2\left(\theta_{3} \vee 1\right)}\right|^{2} \\
& \leq C_{T} e^{-\frac{\beta_{1}}{2}(\sigma-r)}\left(1+|x|^{4\left(\theta_{2} \vee \theta_{3} \vee 1\right)}+|y|^{4\left(\theta_{3} \vee 1\right)}\right) .
\end{aligned}
$$

Hence

$$
\begin{aligned}
\mathbb{E}\left|\frac{1}{\Lambda} \int_{0}^{\Lambda} b_{1}\left(s, x, v_{r}^{s, x, y}\right) d r-\bar{b}_{1}(s, x)\right|^{2} & \leq \frac{C}{\Lambda^{2}}\left(1+|x|^{4\left(\theta_{2} \vee \theta_{3} \vee 1\right)}+|y|^{4\left(\theta_{3} \vee 1\right)}\right) \int_{0}^{\Lambda} \int_{r}^{\Lambda} e^{-\frac{\beta_{1}}{2}(\sigma-r)} d \sigma d r \\
& \leq \frac{C}{\Lambda}\left(1+|x|^{4\left(\theta_{2} \vee \theta_{3} \vee 1\right)}+|y|^{4\left(\theta_{3} \vee 1\right)}\right)
\end{aligned}
$$

The proof is completed.

Lemma 4.6. Assume that the conditions (A1)-(A5) hold. Then, for any $t \in[0, T]$, we have

$$
\mathbb{E}\left\|\int_{0}^{t} b_{1}\left(r\left(\delta_{\epsilon}\right), u_{r\left(\delta_{\epsilon}\right)}^{\epsilon}, \hat{v}_{r}^{\epsilon}\right)-\bar{b}_{1}\left(r\left(\delta_{\epsilon}\right), u_{r\left(\delta_{\epsilon}\right)}^{\epsilon}\right) d r\right\|_{\alpha}^{2} \leq C_{\alpha, x, y, T}\left(\frac{\epsilon}{\delta_{\epsilon}}+\delta_{\epsilon}^{\frac{1}{2}-\alpha}\right) .
$$

Proof: By elementary inequality, we have

$$
\begin{gathered}
\mathbb{E}\left\|\int_{0}^{t} b_{1}\left(r\left(\delta_{\epsilon}\right), u_{r\left(\delta_{\epsilon}\right)}^{\epsilon}, \hat{v}_{r}^{\epsilon}\right)-\bar{b}_{1}\left(r\left(\delta_{\epsilon}\right), u_{r\left(\delta_{\epsilon}\right)}^{\epsilon}\right) d r\right\|_{\alpha}^{2} \\
\leq 3 \mathbb{E}\left|\sum_{k=0}^{\left\lfloor t / \delta_{\epsilon}\right\rfloor-1} \int_{k \delta_{\epsilon}}^{(k+1) \delta_{\epsilon}} b_{1}\left(k \delta_{\epsilon}, u_{k \delta_{\epsilon}}^{\epsilon}, \hat{v}_{r}^{\epsilon}\right)-\bar{b}_{1}\left(k \delta_{\epsilon}, u_{k \delta_{\epsilon}}^{\epsilon}\right) d r\right|^{2}
\end{gathered}
$$




$$
\begin{aligned}
& +3 \mathbb{E}\left|\int_{\left\lfloor t / \delta_{\epsilon}\right\rfloor \delta_{\epsilon}}^{t} b_{1}\left(r\left(\delta_{\epsilon}\right), u_{r\left(\delta_{\epsilon}\right)}^{\epsilon}, \hat{v}_{r}^{\epsilon}\right)-\bar{b}_{1}\left(r\left(\delta_{\epsilon}\right), u_{r\left(\delta_{\epsilon}\right)}^{\epsilon}\right) d r\right|^{2} \\
& +3 \mathbb{E}\left|\int_{0}^{t}(t-r)^{-\alpha-1}\right| \int_{r}^{t} b_{1}\left(s\left(\delta_{\epsilon}\right), u_{s\left(\delta_{\epsilon}\right)}^{\epsilon}, \hat{v}_{s}^{\epsilon}\right)-\bar{b}_{1}\left(s\left(\delta_{\epsilon}\right), u_{s\left(\delta_{\epsilon}\right)}^{\epsilon}\right) d s|d r|^{2} \\
& :=\mathcal{K}_{t}^{1}+\mathcal{K}_{t}^{2}+\mathcal{K}_{t}^{3} .
\end{aligned}
$$

According to Lemma 4.5 and Lemma 4.3, we can get

$$
\begin{aligned}
& \mathcal{K}_{t}^{1} \leq 3\left\lfloor t / \delta_{\epsilon}\right\rfloor \sum_{k=0}^{\left\lfloor t / \delta_{\epsilon}\right\rfloor-1} \mathbb{E}\left|\int_{k \delta_{\epsilon}}^{(k+1) \delta_{\epsilon}} b_{1}\left(k \delta_{\epsilon}, u_{k \delta_{\epsilon}}^{\epsilon}, \hat{v}_{r}^{\epsilon}\right)-\bar{b}_{1}\left(k \delta_{\epsilon}, u_{k \delta_{\epsilon}}^{\epsilon}\right) d r\right|^{2} \\
& \leq \frac{C_{T}}{\delta_{\epsilon}^{2}} \max _{0 \leq k \leq\left\lfloor t / \delta_{\epsilon}\right\rfloor-1} \mathbb{E}\left|\int_{0}^{\delta_{\epsilon}} b_{1}\left(k \delta_{\epsilon}, u_{k \delta_{\epsilon}}^{\epsilon}, \hat{v}_{k \delta_{\epsilon}+r}^{\epsilon}\right)-\bar{b}_{1}\left(k \delta_{\epsilon}, u_{k \delta_{\epsilon}}^{\epsilon}\right) d r\right|^{2} \\
& \leq C_{T} \frac{\epsilon^{2}}{\delta_{\epsilon}^{2}} \max _{0 \leq k \leq\left\lfloor t / \delta_{\epsilon}\right\rfloor-1} \mathbb{E}\left|\int_{0}^{\delta_{\epsilon} / \epsilon} b_{1}\left(k \delta_{\epsilon}, u_{k \delta_{\epsilon}}^{\epsilon}, \hat{v}_{k \delta_{\epsilon}+\epsilon r}^{\epsilon}\right)-\bar{b}_{1}\left(k \delta_{\epsilon}, u_{k \delta_{\epsilon}}^{\epsilon}\right) d r\right|^{2} \\
& \leq C_{T} \max _{0 \leq k \leq\left\lfloor t / \delta_{\epsilon}\right\rfloor-1} \mathbb{E}\left|\frac{1}{\delta_{\epsilon} / \epsilon} \int_{0}^{\delta_{\epsilon} / \epsilon} b_{1}\left(k \delta_{\epsilon}, u_{k \delta_{\epsilon}}^{\epsilon}, Y_{r}^{k \delta_{\epsilon}, u_{k \delta_{\epsilon}}^{\epsilon}, v_{k \delta_{\epsilon}}^{\epsilon}}\right)-\bar{b}_{1}\left(k \delta_{\epsilon}, u_{k \delta_{\epsilon}}^{\epsilon}\right) d r\right|^{2} \\
& \leq C_{\alpha, x, y, T} \frac{\epsilon}{\delta_{\epsilon}} \text {. }
\end{aligned}
$$

The last equation is thanks to the distribution of $\left(u_{k \delta_{\epsilon}}^{\epsilon}, \hat{v}_{k \delta_{\epsilon}+r}^{\epsilon}\right)$ coincides with the distribution of $\left(u_{k \delta_{\epsilon}}^{\epsilon}, Y_{r / \epsilon}^{\left.k \delta_{\epsilon}, u_{k \delta_{\epsilon}}^{\epsilon}, v_{k \delta_{\epsilon}}^{\epsilon}\right)}\right)$ in the interval $r \in\left[0, \delta_{\epsilon}\right)[15]$, where $Y_{r}^{k \delta_{\epsilon}, u_{k \delta_{\epsilon}}^{\epsilon}, v_{k \delta_{\epsilon}}^{\epsilon}}$ is the solution of the fast equation (1.3) by fixed $k \delta_{\epsilon}>0$, frozen slow component $u_{k \delta_{\epsilon}}^{\epsilon}$ and with initial datum $v_{k \delta_{\epsilon}}^{\epsilon}$, and noise $W^{2}$ independent of both of them.

For $\mathcal{K}_{t}^{2}$, thanks to $(4.7),(4.10)$ and $(4.14)$, we obtain

$$
\mathcal{K}_{t}^{2} \leq C \delta_{\epsilon} \int_{\left\lfloor t / \delta_{\epsilon}\right\rfloor \delta_{\epsilon}}^{t} \mathbb{E}\left|b_{1}\left(r\left(\delta_{\epsilon}\right), u_{r\left(\delta_{\epsilon}\right)}^{\epsilon}, \hat{v}_{r}^{\epsilon}\right)\right|^{2}+\mathbb{E}\left|\bar{b}_{1}\left(r\left(\delta_{\epsilon}\right), u_{r\left(\delta_{\epsilon}\right)}^{\epsilon}\right)\right|^{2} d r \quad \leq C_{\alpha, x, y, T} \delta_{\epsilon}^{2}
$$

Then, using the Hölder inequality for $\mathcal{K}_{t}^{3}$, we have

$$
\begin{aligned}
\mathcal{K}_{t}^{3} \leq & 3 \int_{0}^{t}(t-r)^{-\alpha-\frac{1}{2}} d r \int_{0}^{t}(t-r)^{-\alpha-\frac{3}{2}} \mathbb{E}\left|\int_{r}^{t} b_{1}\left(s\left(\delta_{\epsilon}\right), u_{s\left(\delta_{\epsilon}\right)}^{\epsilon}, \hat{v}_{s}^{\epsilon}\right)-\bar{b}_{1}\left(s\left(\delta_{\epsilon}\right), u_{s\left(\delta_{\epsilon}\right)}^{\epsilon}\right) d s\right|^{2} d r \\
\leq & C_{\alpha, T} \int_{0}^{\left(\left\lfloor\frac{t}{\delta_{\epsilon}}\right\rfloor-3\right) \delta_{\epsilon}}(t-r)^{-\alpha-\frac{3}{2}} \mathbb{E}\left|\int_{r}^{t} b_{1}\left(s\left(\delta_{\epsilon}\right), u_{s\left(\delta_{\epsilon}\right)}^{\epsilon}, \hat{v}_{s}^{\epsilon}\right)-\bar{b}_{1}\left(s\left(\delta_{\epsilon}\right), u_{s\left(\delta_{\epsilon}\right)}^{\epsilon}\right) d s\right|^{2} d r \\
& +C_{\alpha, T} \int_{\left(\left\lfloor\frac{t}{\delta_{\epsilon}}\right\rfloor-3\right) \delta_{\epsilon}}^{t}(t-r)^{-\alpha-\frac{3}{2}} \mathbb{E}\left|\int_{r}^{t} b_{1}\left(s\left(\delta_{\epsilon}\right), u_{s\left(\delta_{\epsilon}\right)}^{\epsilon}, \hat{v}_{s}^{\epsilon}\right)-\bar{b}_{1}\left(s\left(\delta_{\epsilon}\right), u_{s\left(\delta_{\epsilon}\right)}^{\epsilon}\right) d s\right|^{2} d r \\
:= & \mathcal{K}_{t}^{31}+\mathcal{K}_{t}^{32} .
\end{aligned}
$$

Using the same argument as the proof of (4.26), (4.27) and the fact $\left\lfloor\lambda_{1}\right\rfloor-\left\lfloor\lambda_{2}\right\rfloor \leq \lambda_{1}-\lambda_{2}+1$. Then, thanks to Lemma 4.5, we can get

$\mathcal{K}_{t}^{31} \leq C_{\alpha, T} \int_{0}^{\left(\left\lfloor\frac{t}{\delta_{\epsilon}}\right\rfloor-3\right) \delta_{\epsilon}}(t-r)^{-\alpha-\frac{3}{2}} \mathbb{E}\left|\int_{r}^{\left(\left\lfloor\frac{r}{\delta_{\epsilon}}\right\rfloor+1\right) \delta_{\epsilon}} b_{1}\left(s\left(\delta_{\epsilon}\right), u_{s\left(\delta_{\epsilon}\right)}^{\epsilon}, \hat{v}_{r}^{\epsilon}\right)-\bar{b}_{1}\left(s\left(\delta_{\epsilon}\right), u_{s\left(\delta_{\epsilon}\right)}^{\epsilon}\right) d s\right|^{2} d r$ 


$$
\begin{aligned}
& +C_{\alpha, T} \int_{0}^{\left(\left\lfloor\frac{t}{\delta_{\epsilon}}\right\rfloor-3\right) \delta_{\epsilon}}(t-r)^{-\alpha-\frac{3}{2}} \mathbb{E}\left|\int_{\left(\left\lfloor\frac{r}{\delta_{\epsilon}}\right\rfloor+1\right) \delta_{\epsilon}}^{\left\lfloor\frac{t}{\delta_{\epsilon}}\right\rfloor \delta_{\epsilon}} b_{1}\left(s\left(\delta_{\epsilon}\right), u_{s\left(\delta_{\epsilon}\right)}^{\epsilon}, \hat{v}_{s}^{\epsilon}\right)-\bar{b}_{1}\left(s\left(\delta_{\epsilon}\right), u_{s\left(\delta_{\epsilon}\right)}^{\epsilon}\right) d s\right|^{2} d r \\
& +C_{\alpha, T} \int_{0}^{\left(\left\lfloor\frac{t}{\delta_{\epsilon}}\right\rfloor-3\right) \delta_{\epsilon}}(t-r)^{-\alpha-\frac{3}{2}} \mathbb{E}\left|\int_{\left\lfloor\frac{t}{\delta_{\epsilon}}\right\rfloor \delta_{\epsilon}}^{t} b_{1}\left(s\left(\delta_{\epsilon}\right), u_{s\left(\delta_{\epsilon}\right)}^{\epsilon}, \hat{v}_{s}^{\epsilon}\right)-\bar{b}_{1}\left(s\left(\delta_{\epsilon}\right), u_{s\left(\delta_{\epsilon}\right)}^{\epsilon}\right) d s\right|^{2} d r \\
& \leq C_{\alpha, T} \int_{0}^{\left(\left\lfloor\frac{t}{\delta_{\epsilon}}\right\rfloor-3\right) \delta_{\epsilon}}\left(t-\left(\left\lfloor t / \delta_{\epsilon}\right\rfloor-3\right) \delta_{\epsilon}\right)^{-\alpha-\frac{3}{2}}\left(\left(\left\lfloor r / \delta_{\epsilon}\right\rfloor+1\right) \delta_{\epsilon}-r\right) \\
& \times \int_{r}^{\left(\left\lfloor\frac{r}{\delta_{\epsilon}}\right\rfloor+1\right) \delta_{\epsilon}} \mathbb{E}\left|b_{1}\left(s\left(\delta_{\epsilon}\right), u_{s\left(\delta_{\epsilon}\right)}^{\epsilon}, \hat{v}_{s}^{\epsilon}\right)-\bar{b}_{1}\left(s\left(\delta_{\epsilon}\right), u_{s\left(\delta_{\epsilon}\right)}^{\epsilon}\right)\right|^{2} d s d r \\
& +C_{\alpha, T} \int_{0}^{\left(\left\lfloor\frac{t}{\delta_{\epsilon}}\right\rfloor-3\right) \delta_{\epsilon}}(t-r)^{-\alpha-\frac{3}{2}}\left(\left\lfloor t / \delta_{\epsilon}\right\rfloor-\left\lfloor r / \delta_{\epsilon}\right\rfloor-1\right) \\
& \times \sum_{k=\left\lfloor\frac{r}{\delta_{\epsilon}}\right\rfloor+1}^{\left\lfloor\frac{t}{\delta_{\epsilon}}\right\rfloor-1} \mathbb{E}\left|\int_{k \delta_{\epsilon}}^{(k+1) \delta_{\epsilon}} b_{1}\left(k \delta_{\epsilon}, u_{k \delta_{\epsilon}}^{\epsilon}, \hat{v}_{s}^{\epsilon}\right)-\bar{b}_{1}\left(k \delta_{\epsilon}, u_{k \delta_{\epsilon}}^{\epsilon}\right) d s\right|^{2} d r \\
& +C_{\alpha, T} \int_{0}^{\left(\left\lfloor\frac{t}{\delta_{\epsilon}}\right\rfloor-3\right) \delta_{\epsilon}}\left(t-\left(\left\lfloor t / \delta_{\epsilon}\right\rfloor-3\right) \delta_{\epsilon}\right)^{-\alpha-\frac{3}{2}}\left(t-\left\lfloor t / \delta_{\epsilon}\right\rfloor \delta_{\epsilon}\right) \\
& \times \int_{\left\lfloor\frac{t}{\delta_{\epsilon}}\right\rfloor \delta_{\epsilon}}^{t} \mathbb{E}\left|b_{1}\left(s\left(\delta_{\epsilon}\right), u_{s\left(\delta_{\epsilon}\right)}^{\epsilon}, \hat{v}_{s}^{\epsilon}\right)-\bar{b}_{1}\left(s\left(\delta_{\epsilon}\right), u_{s\left(\delta_{\epsilon}\right)}^{\epsilon}\right)\right|^{2} d s d r \\
& \leq C_{\alpha, x, y, T} \int_{0}^{\left(\left\lfloor\frac{t}{\delta_{\epsilon}}\right\rfloor-3\right) \delta_{\epsilon}}\left(4 \delta_{\epsilon}\right)^{-\alpha-\frac{3}{2}} \delta_{\epsilon}^{2} d r+C_{\alpha, T} \int_{0}^{\left(\left\lfloor\frac{t}{\delta_{\epsilon}}\right\rfloor-3\right) \delta_{\epsilon}}(t-r)^{-\alpha-\frac{3}{2}}\left(\left\lfloor t / \delta_{\epsilon}\right\rfloor-\left\lfloor r / \delta_{\epsilon}\right\rfloor-1\right)^{2} \\
& \times \max _{\left\lfloor\frac{r}{\delta_{\epsilon}}\right\rfloor+1 \leq k \leq\left\lfloor\frac{t}{\delta_{\epsilon}}\right\rfloor-1} \mathbb{E}\left|\int_{k \delta_{\epsilon}}^{(k+1) \delta_{\epsilon}} b_{1}\left(k \delta_{\epsilon}, u_{k \delta_{\epsilon}}^{\epsilon}, \hat{v}_{s}^{\epsilon}\right)-\bar{b}_{1}\left(k \delta_{\epsilon}, u_{k \delta_{\epsilon}}^{\epsilon}\right) d s\right|^{2} d r \\
& \leq \frac{C_{\alpha, T}}{\delta_{\epsilon}^{2}} \int_{0}^{t}(t-r)^{\frac{1}{2}-\alpha} d r \max _{0 \leq k \leq\left\lfloor\frac{t}{\delta_{\epsilon}}\right\rfloor-1} \mathbb{E}\left|\int_{k \delta_{\epsilon}}^{(k+1) \delta_{\epsilon}} b_{1}\left(k \delta_{\epsilon}, u_{k \delta_{\epsilon}}^{\epsilon}, \hat{v}_{s}^{\epsilon}\right)-\bar{b}_{1}\left(k \delta_{\epsilon}, u_{k \delta_{\epsilon}}^{\epsilon}\right) d s\right|^{2} \\
& +C_{\alpha, x, y, T} \delta_{\epsilon}^{\frac{1}{2}-\alpha} \\
& \leq C_{\alpha, x, y, T}\left(\delta_{\epsilon}^{\frac{1}{2}-\alpha}+\epsilon / \delta_{\epsilon}\right) \text {. }
\end{aligned}
$$

For $\mathcal{K}_{t}^{32}$, using the assumption (A2) and thanks to (4.10) and (4.14), it yields

$$
\begin{aligned}
\mathcal{K}_{t}^{32} & \leq C_{\alpha, T} \int_{\left(\left\lfloor\frac{t}{\delta_{\epsilon}}\right\rfloor-3\right) \delta_{\epsilon}}^{t}(t-r)^{-\alpha-\frac{1}{2}} \int_{r}^{t} \mathbb{E}\left|b_{1}\left(s\left(\delta_{\epsilon}\right), u_{s\left(\delta_{\epsilon}\right)}^{\epsilon}, \hat{v}_{s}^{\epsilon}\right)-\bar{b}_{1}\left(s\left(\delta_{\epsilon}\right), u_{s\left(\delta_{\epsilon}\right)}^{\epsilon}\right)\right|^{2} d s d r \\
& \leq C_{\alpha, x, y, T} \int_{\left(\left\lfloor\frac{t}{\delta_{\epsilon}}\right\rfloor-3\right) \delta_{\epsilon}}^{t}(t-r)^{\frac{1}{2}-\alpha} d r \leq C_{\alpha, x, y, T} \delta_{\epsilon}^{\frac{3}{2}-\alpha} .
\end{aligned}
$$

Hence

$$
\mathcal{K}_{t}^{3} \leq C_{\alpha, x, y, T}\left(\delta_{\epsilon}^{\frac{1}{2}-\alpha}+\epsilon / \delta_{\epsilon}+\delta_{\epsilon}^{\frac{3}{2}-\alpha}\right)
$$

Substituting (4.26)-(4.28) into (4.25), it follows that (4.24) holds. The proof is completed. $\square$ 
Thanks to (4.21), (4.22) and Lemma 4.6, it follows that

$$
\begin{aligned}
\mathcal{I}_{t}^{1} \leq & C_{\alpha, R, T} \int_{0}^{t}(t-r)^{-2 \alpha} \mathbb{E}\left[\left\|u_{r}^{\epsilon}-\bar{u}_{r}\right\|_{\alpha}^{2} \mathbf{1}_{\Omega_{M} \cap\left\{T \leq \tau_{R}^{\epsilon}\right\}}\right] d r \\
& +C_{\alpha, x, y, R, T}\left(\delta_{\epsilon}^{2 \kappa \wedge 2 \iota \wedge(1 / 2-\alpha)}+\epsilon / \delta_{\epsilon}\right) .
\end{aligned}
$$

Then, substituting (4.18) and (4.29) into (4.17), it yields

$$
\begin{aligned}
\mathbb{E}\left[\left\|u_{t}^{\epsilon}-\bar{u}_{t}\right\|_{\alpha}^{2} \mathbf{1}_{\Omega_{M} \cap\left\{T \leq \tau_{R}^{\epsilon}\right\}}\right] \leq & C_{\alpha, R, M, T} \int_{0}^{t}(t-r)^{-\frac{1}{2}-\alpha} r^{-\frac{1}{2}-\alpha} \mathbb{E}\left[\left\|u_{r}^{\epsilon}-\bar{u}_{r}\right\|_{\alpha}^{2} \mathbf{1}_{\Omega_{M} \cap\left\{T \leq \tau_{R}^{\epsilon}\right\}}\right] d r \\
& +C_{\alpha, R, x, y, T}\left(\delta_{\epsilon}^{2 \kappa \wedge 2 \iota \wedge(1 / 2-\alpha)}+\epsilon / \delta_{\epsilon}\right) .
\end{aligned}
$$

According to the Gronwall-type lemma (Lemma 7.6 in [20]), we deduce (4.16). The proof is completed.

Finally, the proof of our main result can be finished.

Proof of Theorem 2.5: Selecting $\delta_{\epsilon}=\epsilon \ln ^{\epsilon^{-\varsigma}}(0<\varsigma<1)$, we can get

$$
\lim _{\epsilon \rightarrow 0} \sup _{t \in[0, T]} \mathbb{E}\left[\left\|u_{t}^{\epsilon}-\bar{u}_{t}\right\|_{\alpha}^{2} \mathbf{1}_{\Omega_{M} \cap\left\{T \leq \tau_{R}^{\epsilon}\right\}}\right]=0 .
$$

Then, let $M \rightarrow+\infty$, as $\Omega_{M} \nearrow \Omega$, it yields

$$
\lim _{\epsilon \rightarrow 0} \sup _{t \in[0, T]} \mathbb{E}\left[\left\|u_{t}^{\epsilon}-\bar{u}_{t}\right\|_{\alpha}^{2} \mathbf{1}_{\left\{T \leq \tau_{R}^{\epsilon}\right\}}\right]=0
$$

Using the Hölder inequality and Chebushev's inequality, thanks to (4.9), (4.10) and Lemma 7.5 in [20], it yields

$$
\begin{aligned}
& \sup _{t \in[0, T]} \mathbb{E}\left[\left\|u_{t}^{\epsilon}-\bar{u}_{t}\right\|_{\alpha}^{2} \mathbf{1}_{\left\{T>\tau_{R}^{\epsilon}\right\}}\right] \leq \sup _{t \in[0, T]}\left[\mathbb{E}\left\|u_{t}^{\epsilon}-\bar{u}_{t}\right\|_{\alpha}^{4}\right]^{\frac{1}{2}}\left[\mathbb{P}\left(T>\tau_{R}^{\epsilon}\right)\right]^{\frac{1}{2}} \\
\leq & \sup _{t \in[0, T]} R^{-1}\left[\mathbb{E}\left\|u_{t}^{\epsilon}\right\|_{\alpha}^{4}+\mathbb{E}\left\|\bar{u}_{t}\right\|_{\alpha}^{4}\right]^{\frac{1}{2}}\left[\mathbb{E}\left(\sup _{t \in[0, T]}\left\|u_{t}^{\epsilon}\right\|_{\alpha}^{2}+\sup _{t \in[0, T]}\left\|\bar{u}_{t}\right\|_{\alpha}^{2}+\sup _{t \in[0, T]}\left(\Lambda_{\alpha}^{0, t}\left(B^{H}\right)\right)^{2}\right)\right]^{\frac{1}{2}} \\
\leq & C_{\alpha, x, T} R^{-1} .
\end{aligned}
$$

Note that

$$
\sup _{t \in[0, T]} \mathbb{E}\left\|u_{t}^{\epsilon}-\bar{u}_{t}\right\|_{\alpha}^{2}=\sup _{t \in[0, T]} \mathbb{E}\left[\left\|u_{t}^{\epsilon}-\bar{u}_{t}\right\|_{\alpha}^{2} \mathbf{1}_{\left\{T \leq \tau_{R}^{\epsilon}\right\}}\right]+\sup _{t \in[0, T]} \mathbb{E}\left[\left\|u_{t}^{\epsilon}-\bar{u}_{t}\right\|_{\alpha}^{2} \mathbf{1}_{\left\{T>\tau_{R}^{\epsilon}\right\}}\right] .
$$

Thanks to (4.30) and (4.31), let $\epsilon \rightarrow 0$ firstly and $R \rightarrow \infty$ secondly, we can get the desired estimate (2.3). This completes the proof of Theorem 2.5 .

\section{Acknowledgments}

This work was partly supported by the National Natural Science Foundation of China under Grant Nos. 11772255 and 12072264, the National Key Research and Development Program of China under Grant No. 2018AAA0102201, the Fundamental Research Funds for the Central Universities, the Research Funds for Interdisciplinary Subject of Northwestern Polytechnical University, the Shaanxi Project for Distinguished Young Scholars, the Shaanxi Provincial Key R\&D Program 2020KW-013 and 2019TD-010. 


\section{Appendix}

In this section, we give the detailed proofs of Lemma 4.1 and Lemma 4.2:

Proof of Lemma 4.1: According to the estimate (4.1) and the classical Bogoliubov-Krylov argument, it is possible to get that the existence of an invariant measure $\mu^{s, x}$. Then, for any Lipschitz function $\varphi: \mathbb{R}^{m} \rightarrow \mathbb{R}$, thanks to (4.2) and (4.5), we obtain

$$
\begin{aligned}
\left|P_{t}^{s, x} \varphi(y)-\int_{\mathbb{R}^{m}} \varphi(z) \mu^{s, x}(d z)\right| & \leq \int_{\mathbb{R}^{m}}\left|P_{t}^{s, x} \varphi(y)-P_{t}^{s, x} \varphi(z)\right| \mu^{s, x}(d z) \\
& \leq \int_{\mathbb{R}^{m}} \mathbb{E}\left|\varphi\left(v_{t}^{s, x, y}\right)-\varphi\left(v_{t}^{s, x, z}\right)\right| \mu^{s, x}(d z) \\
& \leq L_{\varphi} \int_{\mathbb{R}^{m}} \mathbb{E}\left|v_{t}^{s, x, y}-v_{t}^{s, x, z}\right| \mu^{s, x}(d z) \\
& \leq L_{\varphi} \int_{\mathbb{R}^{m}} e^{-\frac{\beta_{1}}{2} t}|y-z| \mu^{s, x}(d z) \\
& \leq C L_{\varphi} e^{-\frac{\beta_{1}}{2} t}(1+|x|+|y|),
\end{aligned}
$$

where $L_{\varphi}=\sup _{x \neq y} \frac{|\varphi(x)-\varphi(y)|}{|x-y|}$. The invariant measure $\mu^{s, x}$ is unique and strong mixing.

Thanks to (4.1), for all $t>0$, we have

$$
\int_{\mathbb{R}^{m}}|z|^{p} \mu^{s, x}(d z)=\int_{\mathbb{R}^{m}} \mathbb{E}\left|v_{t}^{s, x, z}\right|^{p} \mu^{s, x}(d z) \leq C_{p, T}\left(1+|x|^{p}\right)+\int_{\mathbb{R}^{m}} e^{-\beta_{p}^{*} t}|z|^{p} \mu^{s, x}(d z) .
$$

Therefore, if we take $t>0$ such that $e^{-\beta_{p}^{*} t} \leq 1 / 2$, we can get (4.5). Moreover, due to (4.1), (4.2) and (4.5), we also have

$$
\begin{aligned}
& \left|\mathbb{E} b_{1}\left(s, x, v_{t}^{s, x, y}\right)-\int_{\mathbb{R}^{m}} b_{1}(s, x, z) \mu^{s, x}(d z)\right| \\
= & \left|\int_{\mathbb{R}^{m}} \mathbb{E} b_{1}\left(s, x, v_{t}^{s, x, y}\right)-\mathbb{E} b_{1}\left(s, x, v_{t}^{s, x, z}\right) \mu^{s, x}(d z)\right| \\
\leq & C_{T} \int_{\mathbb{R}^{m}} \mathbb{E}\left[\left|v_{t}^{s, x, y}-v_{t}^{s, x, z}\right|\left(1+|x|^{\theta_{2}}+\left|v_{t}^{s, x, y}\right|^{\theta_{3}}+\left|v_{t}^{s, x, z}\right|^{\theta_{3}}\right)\right] \mu^{s, x}(d z) \\
\leq & C_{T} \int_{\mathbb{R}^{m}}\left[\mathbb{E}\left|v_{t}^{s, x, y}-v_{t}^{s, x, z}\right|^{2} \mathbb{E}\left(1+|x|^{2 \theta_{2}}+\left|v_{t}^{s, x, y}\right|^{2 \theta_{3}}+\left|v_{t}^{s, x, z}\right|^{2 \theta_{3}}\right)\right]^{\frac{1}{2}} \mu^{s, x}(d z) \\
\leq & C_{T} e^{-\frac{\beta_{1}}{2} t} \int_{\mathbb{R}^{m}}|z-y|\left(1+|x|^{\theta_{2}}+|x|^{\theta_{3}}+|y|^{\theta_{3}}+|z|^{\theta_{3}}\right) \mu^{s, x}(d z) \\
\leq & C_{T} e^{-\frac{\beta_{1}}{2} t} \int_{\mathbb{R}^{m}}\left(1+|x|^{\theta_{2} \vee \theta_{3}}+|y|^{\theta_{3} \vee 1}+|z|^{\theta_{3} \vee 1}\right)^{2} \mu^{s, x}(d z) \\
\leq & C_{T} e^{-\frac{\beta_{1}}{2} t}\left(1+|x|^{2\left(\theta_{2} \vee \theta_{3} \vee 1\right)}+|y|^{2\left(\theta_{3} \vee 1\right)}\right) .
\end{aligned}
$$

The proof is completed.

Proof of Lemma 4.2: For any $x_{1}, x_{2} \in \mathbb{R}^{n}$ with $\left|x_{i}\right| \leq R$, thanks to the assumptions and equation (4.1), (4.3) and (4.6), we can get

$$
\left|\bar{b}_{1}\left(s_{1}, x_{1}\right)-\bar{b}_{1}\left(s_{2}, x_{2}\right)\right| \leq\left|\int_{\mathbb{R}^{m}} b_{1}\left(s_{1}, x_{1}, z\right) \mu^{s_{1}, x_{1}}(d z)-\mathbb{E} b_{1}\left(s_{1}, x_{1}, v_{t}^{s_{1}, x_{1}, 0}\right)\right|
$$




$$
\begin{aligned}
& +\left|\mathbb{E} b_{1}\left(s_{1}, x_{1}, v_{t}^{s_{1}, x_{1}, 0}\right)-\mathbb{E} b_{1}\left(s_{2}, x_{1}, v_{t}^{s_{1}, x_{1}, 0}\right)\right| \\
& +\left|\mathbb{E} b_{1}\left(s_{2}, x_{1}, v_{t}^{s_{1}, x_{1}, 0}\right)-\mathbb{E} b_{1}\left(s_{2}, x_{2}, v_{t}^{s_{1}, x_{1}, 0}\right)\right| \\
& +\left|\mathbb{E} b_{1}\left(s_{2}, x_{2}, v_{t}^{s_{1}, x_{1}, 0}\right)-\mathbb{E} b_{1}\left(s_{2}, x_{2}, v_{t}^{s_{2}, x_{2}, 0}\right)\right| \\
& +\left|\mathbb{E} b_{1}\left(s_{2}, x_{2}, v_{t}^{s_{2}, x_{2}, 0}\right)-\int_{\mathbb{R}^{m}} b_{1}\left(s_{2}, x_{2}, z\right) \mu^{s_{2}, x_{2}}(d z)\right| \\
\leq & C_{T} e^{-\frac{\beta_{1}}{2} t}\left(1+\left|x_{1}\right|^{2\left(\theta_{2} \vee \theta_{3} \vee 1\right)}+\left|x_{2}\right|^{2\left(\theta_{2} \vee \theta_{3} \vee 1\right)}\right) \\
& +C_{T}\left|s_{1}-s_{2}\right|^{\kappa}\left(1+\left|x_{1}\right|^{\theta_{2}}+\mathbb{E}\left|v_{t}^{s_{1}, x_{1}, 0}\right|^{\theta_{3}}\right) \\
& +C_{R, T}\left|x_{1}-x_{2}\right|\left(1+\mathbb{E}\left|v_{t}^{s_{1}, x_{1}, 0}\right|^{\theta_{1}}\right) \\
& +C_{T} \mathbb{E}\left[\left|v_{t}^{s_{1}, x_{1}, 0}-v_{t}^{s_{2}, x_{2}, 0}\right|\left(1+\left|x_{2}\right|^{\theta_{2}}+\left|v_{t}^{s_{1}, x_{1}, 0}\right|^{\theta_{3}}+\left|v_{t}^{s_{2}, x_{2}, 0}\right|^{\theta_{3}}\right)\right] \\
\leq & C_{T} e^{-\frac{\beta_{1}}{2} t}\left(1+\left|x_{1}\right|^{2\left(\theta_{2} \vee \theta_{3} \vee 1\right)}+\left|x_{2}\right|^{2\left(\theta_{2} \vee \theta_{3} \vee 1\right)}\right) \\
& +C_{T}\left|s_{1}-s_{2}\right|^{\kappa}\left(1+\left|x_{1}\right|^{\theta_{2}}+\left|x_{1}\right|^{\theta_{3}}\right)+C_{R, T}\left|x_{1}-x_{2}\right|\left(1+\left|x_{1}\right|^{\theta_{1}}\right) \\
& +C_{T}\left(\left|s_{1}-s_{2}\right|^{\iota}+\left|x_{1}-x_{2}\right|\right)\left(1+\left|x_{1}\right|^{2\left(\alpha_{1} \vee \theta_{3}\right)}+\left|x_{2}\right|^{2\left(\alpha_{1} \vee \alpha_{2} \vee \theta_{2} \vee \theta_{3}\right)}\right) \\
\leq & C_{R, T} e^{-\frac{\beta_{1}}{2} t}+C_{R, T}\left(\left|s_{1}-s_{2}\right|^{\kappa}+\left|s_{1}-s_{2}\right|^{\iota}+\left|x_{1}-x_{2}\right|\right) .
\end{aligned}
$$

Let $t \rightarrow+\infty$, we obtain (4.8). Moreover, thanks to (4.5), we also have

$$
\left|\bar{b}_{1}(t, x)\right| \leq \int_{\mathbb{R}^{m}}\left|b_{1}(t, x, z)\right| \mu^{t, x}(d z) \leq C_{T} \int_{\mathbb{R}^{m}}(1+|x|+|z|) \mu^{t, x}(d z) \leq C_{T}(1+|x|) .
$$

The proof is completed.

\section{References}

[1] A. N. Kolmogorov, Wienershe spiralen und einige andere interessante kurven in hilbertschen raum, C. R. (Dokl.) Acad. Sci. URSS (NS) 26 (1940) 115-118.

[2] B. B. Mandelbrot, J. W. Van Ness, Fractional brownian motions, fractional noises and applications, SIAM Review 10 (4) (1968) 422-437.

[3] R. Khasminskii, On the averaging principle for stochastic differential Itô equations, Kybernetika. 4 (1968) 260-279.

[4] D. Givon, Strong convergence rate for two-time-scale jump-diffusion stochastic differential systems, Multiscale. Model. Sim. 6 (2007) 577-594.

[5] M. Freidlin, A. Wentzell, Random Perturbations of Dynamical Systems, Springer Science and Business Media, Berlin Heidelberg, 2012.

[6] J. Q. Duan, W. Wang, Effective Dynamics of Stochastic Partial Differential Equations, Elsevier, 2014.

[7] Y. Xu, J. Q. Duan, W. Xu, An averaging principle for stochastic dynamical systems with Lévy noise, Physica D. 240 (2011) 1395-1401. 
[8] Y. Xu, B. Pei, J. L. Wu, Stochastic averaging principle for differential equations with non-Lipschitz coefficients driven by fractional Brownian motion, Stoch. Dynam. 17 (2017) 1750013.

[9] S. Cerrai, A Khasminskii type averaging principle for stochastic reaction-diffusion equations, Ann. Appl. Probab. 19 (2009) 899-948.

[10] S. Cerrai, Averaging principle for systems of reaction-diffusion equations with polynomial nonlinearities perturbed by multiplicative noise, SIAM. J. Math. Anal. 43 (2011) $2482-2518$.

[11] W. Wang, A. Roberts, Average and deviation for slow-fast stochastic partial differential equations, J. Differ. Equations. 253 (2012) 1265-1286.

[12] B. Pei, Y. Xu, J. L. Wu, Two-time-scales hyperbolic-parabolic equations driven by Poisson random measures: Existence, uniqueness and averaging principles, J. Math. Anal. Appl. 447 (2017) 243-268.

[13] B. Pei, Y. Xu, G. Yin, Averaging principles for SPDEs driven by fractional Brownian motions with random delays modulated by two-time-scale Markov switching processes, Stoch. Dynam. 18 (2018) 1850023.

[14] S. Cerrai, A. Lunardi, Averaging principle for non-autonomous slow-fast systems of stochastic reaction-diffusion equations: The almost periodic case, SIAM. J. Math. Anal. 49 (2017) 2843-2884.

[15] W. Liu, M. Röckner, X. B. Sun, Y. C. Xie, Averaging principle for slow-fast stochastic differential equations with time dependent locally lipschitz coefficients, J. Differ. Equations 268 (6) (2020) 2910-2948.

[16] Y. Xu, R. F. Wang, Averaging principles for non-autonomous two-time-scale stochastic reaction-diffusion equations with jump, Complexity 2020 (2020) 1-22.

[17] J. Guerra, D. Nualart, Stochastic differential equations driven by fractional Brownian motion and standard Brownian motion, Stoch. Anal. Appl. 26 (5) (2008) 1053-1075.

[18] M. Zähle, Integration with respect to fractal functions and stochastic calculus, Prob. Theory Relat. Fields 111 (3) (1998) 333-374.

[19] S. Samko, A. Kilbas, O. Marichev, Fractional integrals and derivatives, theory and applications, Gordon and Breach Science Publishers, Yvendon, 1993.

[20] D. Nualart, A. Răşcanu, Differential equations driven by fractional Brownian motion, Collectanea Mathematica 53 (1) (2002) 55-81.

[21] A. Garsia, E. Rodemich, Monotonicity of certain functionals under rearrangement, Annales Institut Fourier 24 (2) (1974) 67-116.

[22] B. Pei, Y. Inahama, Y. Xu, Averaging principles for mixed fast-slow systems driven by fractional Brownian motion, arXiv preprint arXiv:2001.06945. 